\title{
Conditional GWAS of non-CG transposon methylation in Arabidopsis thaliana reveals major polymorphisms in five genes
}

\author{
Eriko Sasaki ${ }^{1,2^{*}}$, Joanna Gunis ${ }^{1}$, Ilka Reichardt-Gomez ${ }^{1,3}$, Viktoria Nizhynska ${ }^{1}$, Magnus
} Nordborg ${ }^{1 *}$

1. Gregor Mendel Institute of Molecular Plant Biology, Austrian Academy of Sciences, Vienna BioCenter (VBC), Dr. Bohr-Gasse 3, 1030 Vienna, Austria

2. Faculty of Science. Kyushu University 744, Motooka, Nishi-ku, Fukuoka 819-0395, Japan

3. Max Planck Institute of Molecular Cell Biology and Genetics, Dresden, Pfotenhauerstraße 108, 01307 Dresden, Germany

* Corresponding authors: Eriko Sasaki <sasaki.eriko.997@m.kyushu-u.ac.jp>, Magnus Nordborg <magnus.nordborg@gmi.oeaw.ac.at>

\section{Abstract}

Genome-wide association studies (GWAS) have revealed that the striking natural variation for DNA CHH-methylation ( $\mathrm{mCHH} ; \mathrm{H}$ is $\mathrm{A}, \mathrm{T}$, or $\mathrm{C}$ ) of transposons has oligogenic architecture involving major alleles at a handful of known methylation regulators. Here we use a conditional GWAS approach to show that CHG-methylation (mCHG) has a similar genetic architecture - once $\mathrm{mCHH}$ is statistically controlled for. We identify five key trans-regulators that appear to modulate $\mathrm{mCHG}$ levels, and show that they interact with a previously identified modifier of $\mathrm{mCHH}$ in regulating natural transposon mobilization.

\section{Introduction}

Organisms have developed defense systems to protect the genome from transposable elements, which can act as 'selfish genes' and cause considerable damage (Chuong et al., 2017; Deniz et al., 2019). Cytosine DNA methylation is a major component of genome defense that is found in both mammals and plants, albeit with significant differences (Law and Jacobsen, 2010; Deniz et al., 2019). For instance, whereas mammals mostly have CG methylation (mCG), methylation in plants also occurs in the $\mathrm{CHG}$ and $\mathrm{CHH}$ contexts $(\mathrm{H}$ is $\mathrm{A}$, 
$\mathrm{T}$, or $\mathrm{C}$ ). $\mathrm{mCG}$ in plants is known to be maintained through both mitosis and meiosis by DNA METHYLTRANSFERASE 1 (MET1; DNMT1 in humans) - in contrast to $\mathrm{CHH}$ methylation $(\mathrm{mCHH})$, which is re-established after cell division by several pathways, including the RNAdirected DNA methylation (RdDM) pathway and the CHROMOMETHYLASE 2 (CMT2) pathway (Kawashima and Berger, 2014; Matzke et al., 2015). Unlike mCG, which is stably inherited, $\mathrm{mCHH}$ behaves like a molecular phenotype and is strongly influenced by the environment, such as growth temperature (Dubin et al., 2015) and stress (Wibowo et al., 2016). CHG methylation ( $\mathrm{mCHG}$ ) falls somewhere between $\mathrm{mCG}$ and $\mathrm{mCHH}$ in the sense that it can be maintained via positive feedback between CHROMOMETHYLASE3 (CMT3) and KRYPTONITE (KYP), which recognize dimethylation of histone 3 lysine 9 (H3K9me2) and mCHG, respectively (Lindroth et al., 2001; Jackson et al., 2002; Cao and Jacobsen, 2002; Du et al., 2015). Molecular mechanisms aside, the forces shaping variation in DNA methylation remain obscure, and the same is true for their biological significance (Riddle and Richards, 2002; Reinders et al., 2009; Becker et al., 2011; Takuno and Gaut, 2012; Meng et al., 2016; Wibowo et al., 2016; Johannes and Schmitz, 2019; Muyle and Gaut, 2019; Baduel and Colot, 2021). Previously, we demonstrated the existence of large-scale geographic clines for DNA methylation in $A$. thaliana, and used genome-wide association studies (GWAS) to show that $\mathrm{mCHH}$ on transposons is heavily influenced by major polymorphisms at trans-acting modifiers corresponding to known DNA methylation regulators in silencing pathways: CMT2, NUCLEAR RNA POLYMERASE D1B (NRPE1), ARGONAUTE 1 (AGO1), and AGO9 (Dubin et al. 2015, Kawakatsu et al 2016, Sasaki et al 2019). In particular, an allele of NRPE1 (named NRPE1') strongly affects $\mathrm{mCHH}$ levels in RdDM-targeted transposons. NRPE1, which is the largest subunit of RNA polymerase $\mathrm{V}$, localizes to promoter regions of relatively young transposons (Zhong et al., 2012), and Baduel et al. (2021) recently showed that the NRPE1' allele has been associated with recent transposon mobilization, presumably through its effect on DNA methylation. All in all, these findings are suggestive of a highly variable genome defense system.

While both $\mathrm{mCHG}$ and $\mathrm{mCG}$ showed high heritability, GWAS yielded little in terms of significant associations. This might be because these "traits" are highly polygenic, or because they are at least partly transgenerationally inherited, and hence do not behave like standard phenotypes. In this paper we revisit $\mathrm{mCHG}$ variation and use a conditional GWAS approach to reveal that multiple major alleles at trans-acting regulators modify this type of methylation, and are also associated with transposon mobilization. 


\section{Results}

\section{mCHG is strongly correlated with $\mathrm{mCHH}$}

Our starting point is the observation that $\mathrm{mCHG}$ and $\mathrm{mCHH}$ levels on transposons are strongly correlated in the 1001 Epigenomes data set (Kawakatsu et al., 2016), especially for RdDMtargeted transposons (Fig. 1A; see Methods). Much of this variation is due to differences in the environment (including tissue, which can be viewed as a cellular environment), with flower tissue samples showing clear hyper-mCHG compared with leaf samples as expected (Feng et al., 2020; Gutzat et al., 2020). At the same time, variation across individuals is huge even when controlling for known tissue and environmental effects, as observed in the largest leaf sample data set ("Leaf SALK ambient temperature"; $n=846$ ).

Interestingly, the covariance between $\mathrm{mCHH}$ and $\mathrm{mCHG}$ showed the same pattern in data generated by knocking out known or potential DNA methylation regulators in the same genetic background (Fig. 1B) (Stroud et al., 2013). This demonstrates strong co-regulation of these types of methylation, in particular for RdDM-targeted transposons. Loss of RdDM regulation, such as in the double mutant of DOMAINS REARRANGED METHYLASE (DRM) drm1 drm2, causes loss of almost all $\mathrm{mCHH}$ and $\mathrm{mCHG}$ because $\mathrm{mCHG}$ in these regions is mainly established via siRNA (Chan et al., 2006). At the same time, the data demonstrate that some genetic perturbations can affect one type of methylation much more than the other. For example, knocking out CMT3 affects $\mathrm{mCHG}$ levels much more than $\mathrm{mCHH}$ levels.

Based on these observations, it is clear that a substantial fraction of variation in non-CG methylation is due to factors that affect both $\mathrm{mCHH}$ and $\mathrm{mCHG}$. Without replicate measurements, it is difficult to say what fraction of these factors is genetic and what is environmental, but, regardless of this, we hypothesized that the substantial covariance could reduce power of GWAS for either $\mathrm{mCHH}$ or $\mathrm{mCHG}$ (when using a standard univariate model), and that an analysis accounting for this covariance might perform better (Korte et al., 2012; Stegle et al., 2012; Stephens, 2013). In essence, we sought to simplify a complex trait by breaking it into constituent parts (Nilsson-Ehle, 1909; East, 1910; Lande and Arnold, 1983). This insight is the basis for this paper. 

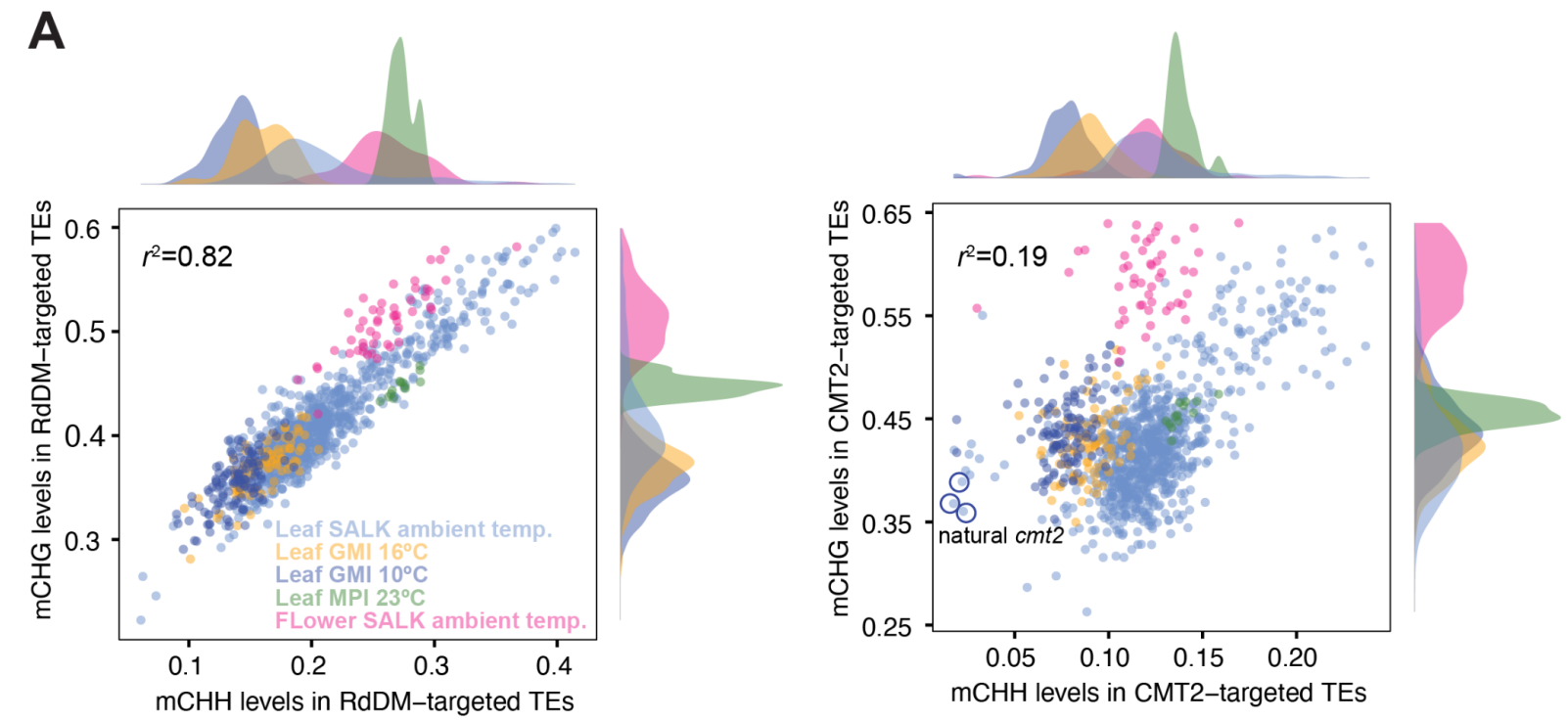

B
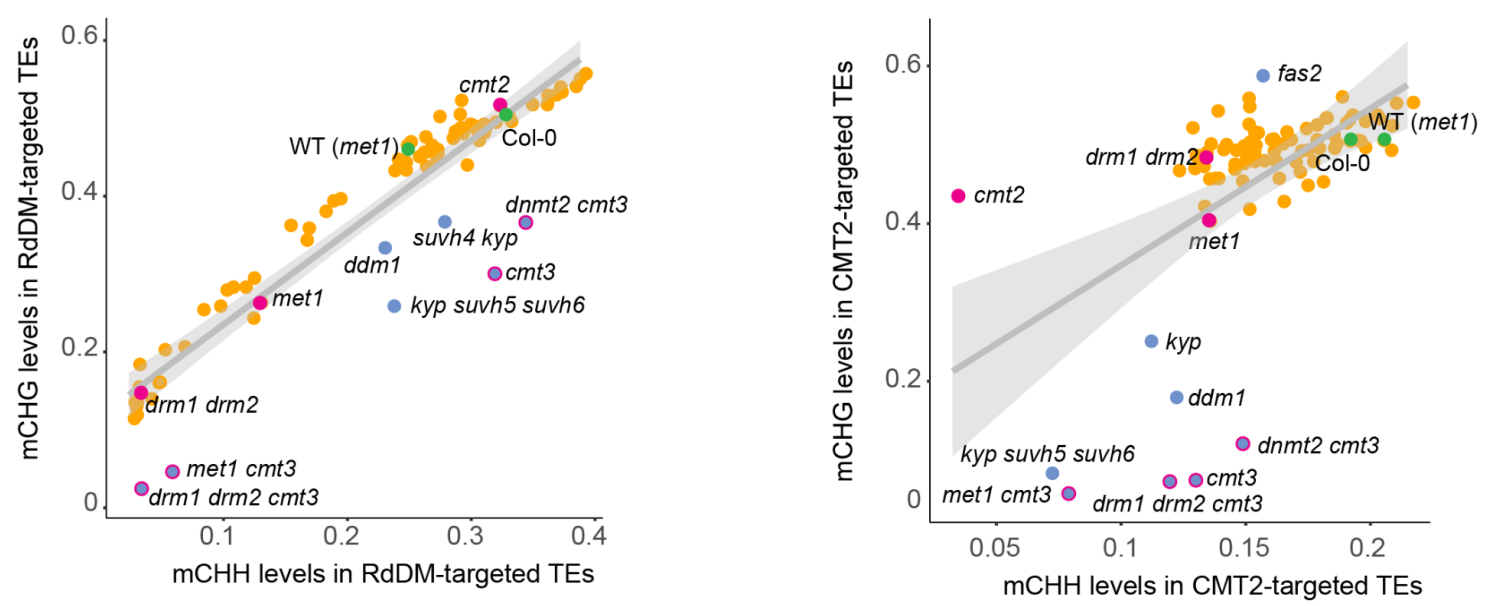

Fig 1. Covariance between $\mathrm{mCHH}$ and $\mathrm{mCHG}$ levels across individuals. (A) Correlation of genomewide average $\mathrm{mCHH}$ and $\mathrm{mCHG}$ levels in RdDM- and CMT2-targeted transposons across 1028 natural inbred lines measured in different conditions (including 79 lines measured in more than one condition; see Kawakatsu et al., 2016) Colors correspond to environments/tissues. Plots on axes show marginal densities. Circled lines carry known natural null alleles of CMT2. (B) The same correlation as in A, but for 85 epigenetics-related loss-of-function mutants (Stroud et al., 2013). Gray areas indicate 99\% confidence intervals around the linear regression lines. Green points denote "wildtypes" (see Stroud et al., 2013); magenta, major DNA methyltransferases. Blue points denote lines with a highly significant effect on $\mathrm{mCHG}$.

\section{Conditional GWAS reveals new associations}

Multi-trait GWAS can be carried out using transformations (e.g., the difference or ratio between traits), conditional approaches in which one or more traits are accounted for as covariates, or full multivariate models (Korte et al., 2012; Stegle et al., 2012; Stephens, 2013). We tried several different approaches, including a multivariate GWAS, but this paper will focus on results from a conditional GWAS of $\mathrm{mCHG}$ with $\mathrm{mCHH}$ as a covariate (denoted $\mathrm{mCHG}_{\mid \mathrm{mCHH}}$ ) 
because this gave the clearest results. Other approaches produced consistent results, and we will briefly discuss them later.

Figure 2 contrasts the performance of univariate and conditional GWAS. As previously noted, univariate GWAS of $\mathrm{mCHG}$ does not yield any significant associations despite moderate SNP-heritability (37\% and 38\% for RdDM- and CMT2-targeted transposons, respectively). As expected given the very strong correlation between $\mathrm{mCHH}$ and $\mathrm{mCHG}$ on RdDM-targeted transposons, the strong associations at AGO1 and NRPE1 found in univariate GWAS of $\mathrm{mCHH}$ (Kawakatsu et al., 2016) give rise to associations for $\mathrm{mCHG}$ as well, but they are not genome-wide significant (AGO1 Chr1:17895231, - $\log _{10} p$-value $=6.51$ and NRPE1 Chr2:16714815, $-\log _{10} p$-value=5.23). The previously identified CMT2-association with $\mathrm{mCHH}$ on CMT2-targeted transposons (Dubin et al., 2015; Kawakatsu et al., 2016; Sasaki et al., 2019; Shen et al., 2014) is not apparent, consistent with the much weaker correlation between $\mathrm{mCHH}$ and $\mathrm{mCHG}$ on these transposons.

The contrast between these univariate GWAS results and the conditional analysis is striking. GWAS for $\mathrm{mCHG}$ while controlling for $\mathrm{mCHH}\left(\mathrm{mCHG}_{\mid \mathrm{mCHH}}\right)$ revealed five clear peaks for RdDM-targeted transposons, three of which were also found for CMT2-targeted transposons. The peaks are above or near genome-wide significance using a conservative threshold (Figs. 2, S1; Table S1). As will be discussed further below, these associations jointly explain $30.5 \%$ and $14.8 \%$ of $\mathrm{mCHG}_{\mid \mathrm{mCHH}}$ in RdDM- and $\mathrm{CMT}_{2}$-targeted transposons, respectively. The previously mentioned AGO1 and NRPE1 associations disappear completely, consistent with their being strongly correlated with $\mathrm{mCHH}$, and hence controlled for. The improved performance of conditional GWAS is also evident from a massive enrichment of associations near a priori candidates (Atwell et al., 2010). With conditional GWAS, we observed an up to 45-fold excess of associations near annotated epigenetic modifiers (Kawakatsu et al., 2016; Sasaki et al., 2019), compared to an 4- to 10-fold excess for the univariate analyses (Fig. 2). 
A
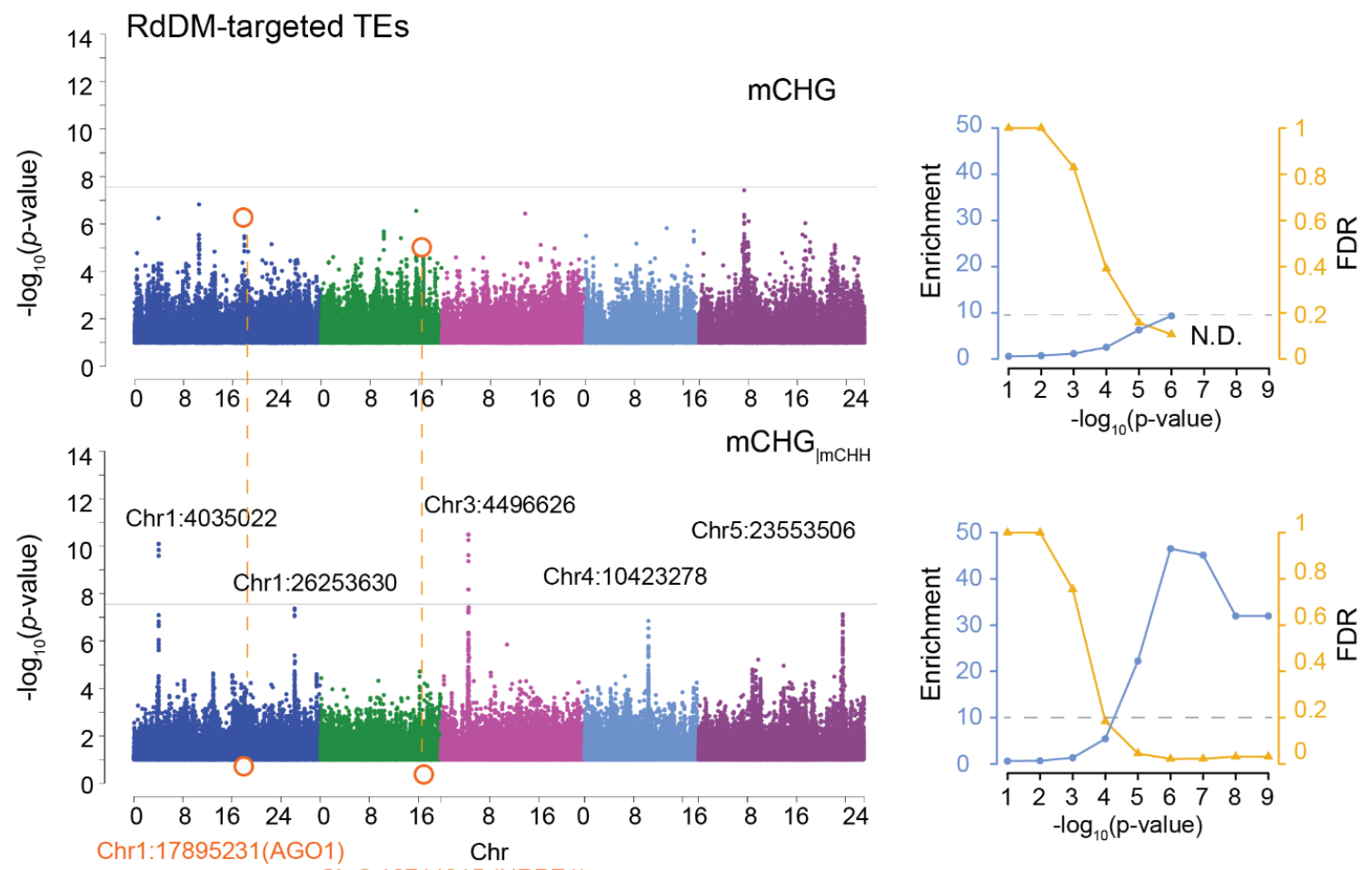

Chr2:16714815 (NRPE1)

B
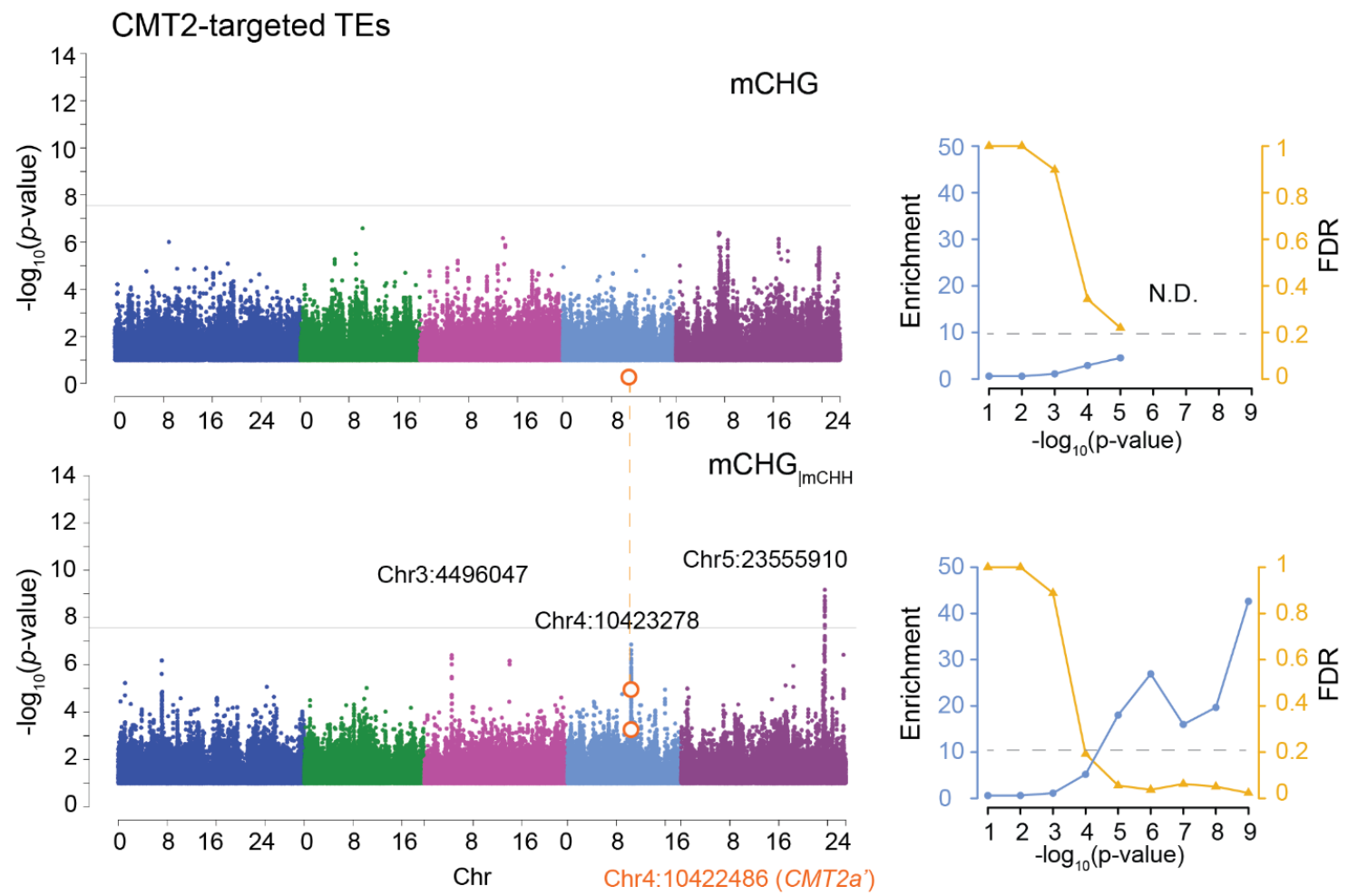

Fig 2. Comparison of univariate and conditional GWAS of mCHG. The analysis was done separately for (A) RdDM-targeted and (B) CMT2-targeted transposons, using the 774 lines in the global panel from the 1001 Epigenomes Project ("SALK leaf in ambient temperature"; see Fig. 1). For each case, the upper Manhattan plot shows univariate GWAS of $\mathrm{mCHG}$ methylation and the lower GWAS of $m \mathrm{mHG}$ controlling for $\mathrm{mCHH}$. Horizontal gray lines show genome-wide significance $(p=0.05$ after Bonferroni-correction). The line plots show enrichment of a priori genes and FDR (see text), with horizontal dashed lines indicating an FDR of $20 \%$. 
The enrichment for a priori candidates also allows us to estimate a False Discovery Rate (FDR) for this set of genes (Atwell et al., 2010). Four of the five major associations can be identified with a priori candidates at very low FDRs (e.g. FDR $<0.05$ using a significance threshold of $-\log _{10} p$-value $>6$; see Fig. 2 ), but it is notable that FDR is low even for associations that are nowhere near genome-wide significance. This is not true for the univariate analyses. For example, at an FDR of $20 \%$, univariate GWAS of $\mathrm{mCHG}$ identifies only AGO1 and NRPE1 for RdDM-targeted transposons, and nothing for CMT2-targeted transposons (Table S2), whereas conditional GWAS of $\mathrm{mCHG}_{\mid \mathrm{mCHH}}$ generates a long list of known regulators of $\mathrm{mCHG}$ (Table S2). For RdDM-targeted transposons, we find four a priori genes above or near genome-wide significance: the previously mentioned CMT2 and CMT3, plus MIR823a, which encodes a microRNA targeting CMT3 (Rajagopalan et al., 2006; Papareddy et al., 2021), and MULTICOPY SUPPRESSOR OF IRA1 (MSI1), likely a component of chromatin assembly factor (CAF-1) responsible for proper heterochromatin formation with FASCIATA1 (FAS1) and FASCIATA2 (FAS2) (Hennig et al., 2003, 2005). At lower significance levels, we find REPRESSOR OF SILENCING 3 (ROS3) and DNA METHYLTRANSFERASE 2 (DNMT2). ROS3 is a DNA demethylase that interacts with REPRESSOR OF SILENCING 1 (ROS1) (Zheng et al., 2008) and DNMT2 is associated with histone deacetylation (Song et al., 2010) and RNA methyltransferase activity (Goll et al. 2006) (and could therefore be a false positive since the effects on DNA methylation is under debate; see Goll et al. 2006; Song et al. 2010). For CMT2-targeted transposons, the list of associated a priori candidates includes four genes also associated with RdDM-targeted transposons, namely MIR823A, CMT2, ROS3, and MSI1, but there are also two specific genes, FAS1 and DECREASED DNA METHYLATION 1 (DDM1), a chromatin-remodeler responsible for heterochromatin formation (Soppe et al., 2002; Osakabe et al., 2021). As shown in Fig. 1B, ddm1 strongly reduces $\mathrm{mCHG}$, and fas2, a functionally redundant FAS1 homolog, increases $\mathrm{CMT2}$-targeted $\mathrm{mCHG}$ in a $\mathrm{mCHH}$ independent manner (Stroud et al., 2013).

\section{Genes underlying major associations}

\section{On searching for causal genes}

Identifying causal genes and mechanisms from GWAS results is notoriously difficult (Gallagher and Chen-Plotkin, 2018). Peaks of association often cover multiple genes - this is certainly true in the gene-dense genome of $A$. thaliana (Atwell et al., 2010) - and functional annotation is of limited use for complex traits with unknown genetic basis. We are in a stronger position, however, because, just like in our previous work on $\mathrm{mCHH}$ (Sasaki et al., 2019), the significant GWAS peaks (Fig. 2) are narrow, and clearly pinpoint genes a priori known to be specifically involved in well-defined molecular phenotypes (Table 1, Fig. 3). Hence we will discuss these candidates, and the indirect evidence supporting their causal role. 
Table 1. Significant conditional GWAS hits for $\mathbf{m C H G}_{\mid \mathrm{mCHH}}$ *

\begin{tabular}{|c|c|c|c|c|}
\hline SNPS & $\begin{array}{c}-\log _{10} \\
(p \text {-value })\end{array}$ & MAC & $\begin{array}{l}\text { Distance from } \\
\text { a priori gene (bp) }\end{array}$ & Genes within $15 \mathrm{~K} b p^{*}$ \\
\hline \multicolumn{5}{|c|}{$m C H G_{\mid m C H H}$ in RdDM-targeted transposons } \\
\hline Chr1:4035022 & 10.1 & 77 & NA & $\begin{array}{l}\text { AT1G11930, AT1G11940, AT1G11950 } \\
\text { (JMJ26), AT1G11960 }\end{array}$ \\
\hline Chr1:26253630 & 7.38 & 290 & 50 & AT1G69770 (CMT3) \\
\hline Chr3:4496626 & 10.48 & 207 & 196 & $\begin{array}{l}\text { AT3G13700, AT3G13710 (PRA1.F4), } \\
\text { AT3G13720 (PRA1.F3), AT3G13724 } \\
\text { (MIR823A), AT3G13730 (SYP90D1) }\end{array}$ \\
\hline Chr4:10423278 & 6.85 & 301 & 2069 & $\begin{array}{l}\text { AT4G19020 (CMT2), AT4G19030 (NLM1), } \\
\text { AT4G19035 (LCR7) }\end{array}$ \\
\hline Chr5:23553506 & 7.14 & 99 & 2506 & $\begin{array}{l}\text { AT5G58200 (ECT10), AT5G58200, } \\
\text { AT5G58210, AT5G58220 (TTL), } \\
\text { AT5G58230 (MSI1), AT5G58240 (FHIT) }\end{array}$ \\
\hline \multicolumn{5}{|c|}{$m C H G_{\mid m C H H}$ in CMT2-targeted transposons } \\
\hline Chr3:4496047 & 6.42 & 210 & 775 & $\begin{array}{l}\text { AT3G13700, AT3G13710 (PRA1.F4), } \\
\text { AT3G13720 (PRA1.F3), AT3G13724 } \\
\text { (MIR823A), AT3G13730 (SYP90D1) }\end{array}$ \\
\hline Chr4:10423278 & 6.86 & 301 & 2069 & $\begin{array}{l}\text { AT4G19020 (CMT2), AT4G19030 (NLM1), } \\
\text { AT4G19035 (LCR7) }\end{array}$ \\
\hline Chr5:23555910 & 9.17 & 64 & 102 & $\begin{array}{l}\text { AT5G58200, AT5G58210, AT5G58220 } \\
(\text { TTL), AT5G58230 (MSI1), AT5G58240 } \\
(\text { FHIT), AT5G58250, AT5G58260 (NdhN) }\end{array}$ \\
\hline
\end{tabular}

${ }^{*}$ Bold denotes a priori epigenetic regulators 
A
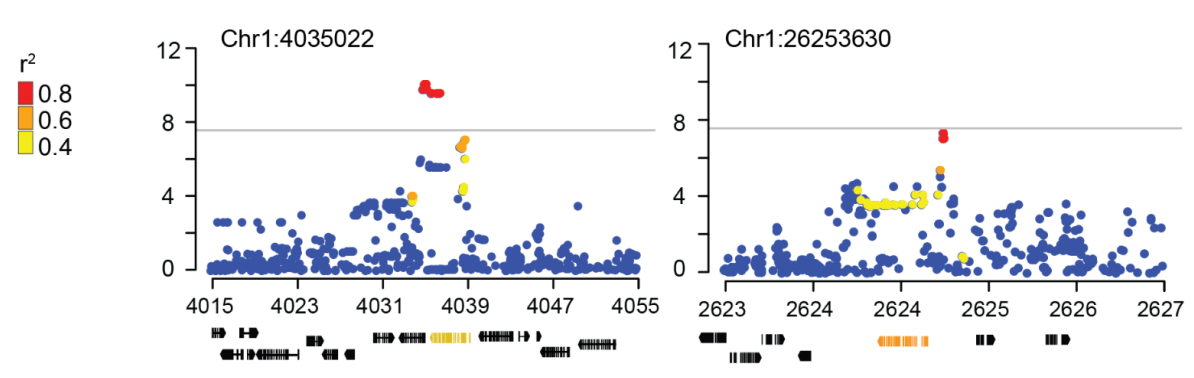

AT1G11950 (JMJ26)

AT1G69770 (CMT3)

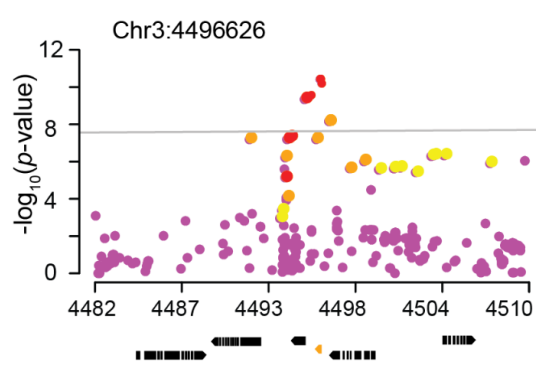

AT3G13724 (MIR823A)

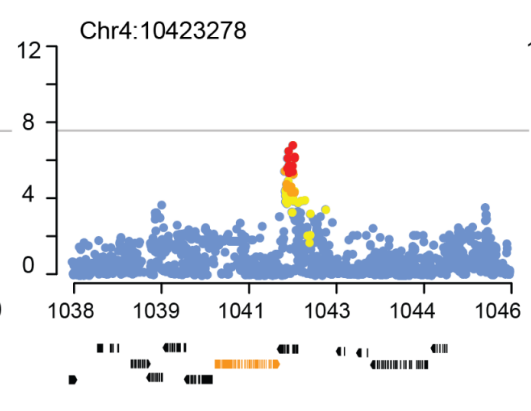

AT4G19020 (CMT2)

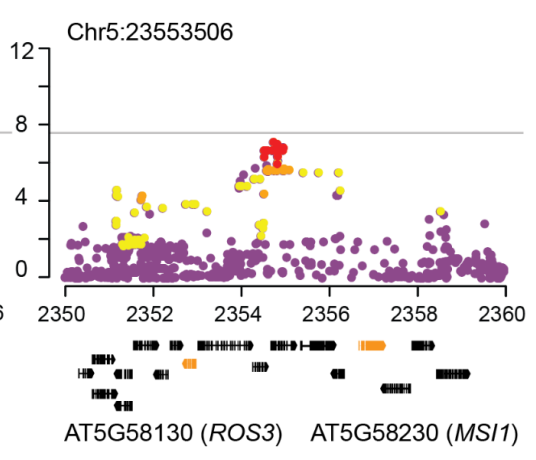

B

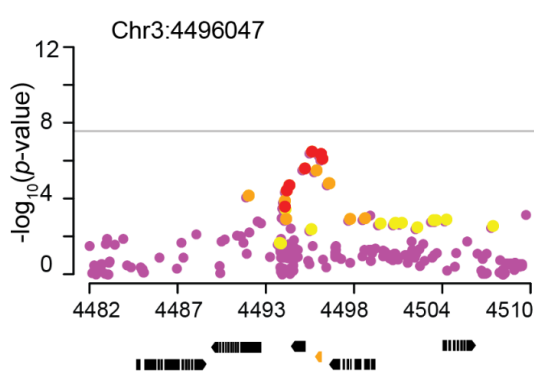

AT3G13724 (MIR823A)

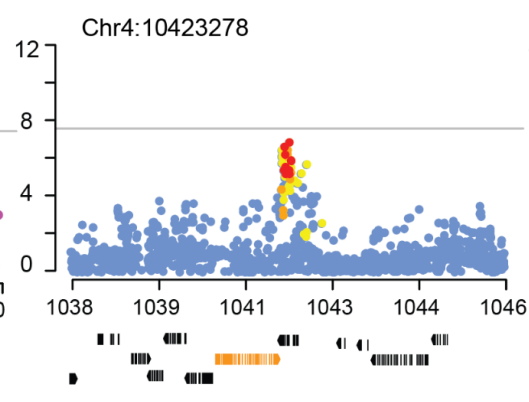

AT4G19020 (CMT2)

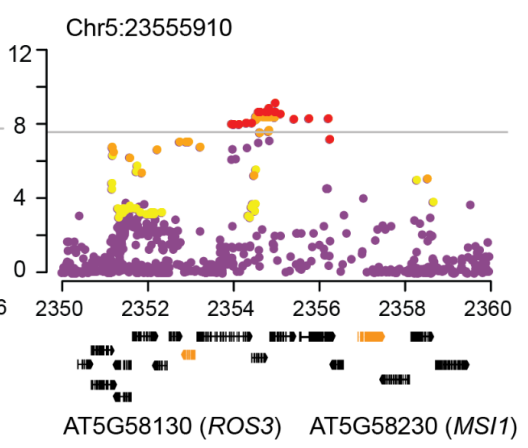

Fig 3. Candidate loci underlying $\mathbf{m C H G}_{\mid \mathbf{m c H H}}$ variation. Zoomed-in Manhattan plots aroundconditional GWAS peaks in Fig. 2 for (A) RdDM- and (B) CMT2-targeted transposons. Gene models below the plots show the candidate genes in orange (see Table 1). Colored dots represent SNPs in strong linkage disequilibrium with top SNPs.

\section{Multi-layered direct CMT3 regulation affects $\mathrm{mCHG}$ variation}

Based on mutant phenotypes, CMT3 is a strong a priori candidate for regulating $\mathrm{mCHG}$ (Fig. 1). Consistent with this, one of the five major associations from $\mathrm{GWAS}_{\mathrm{S}} \mathrm{mCHG} \mathrm{mCHH}_{\mathrm{m}}$ pinpoints CMT3 and another MIR823a, a gene encoding a microRNA that directly down-regulates CMT3 by cleaving its transcripts during early embryogenesis (Papareddy et al., 2021). The most significant peak for $\mathrm{mCHG}_{\mid \mathrm{mCHH}}$ on RdDM-targeted transposons was located $196 \mathrm{bp}$ downstream of MIR823a, while the peak corresponding to CMT3 was located 50 bp upstream of the gene (Fig. 4A, Table 1). GWAS for CMT2-targeted transposons also pinpointed MIR823a, although the association is weaker, and the most significant SNP further away (Fig. 
3B, Table 1). This association was also recently found by Hüther et al. (2022) using GWAS for unconditional $\mathrm{mCHG}$ levels of individual transposons.

The MIR823A polymorphism appears to almost exclusively affect mCHG (Figs. S2, S3), primarily targeting the same transposons as a CMT3 knock-out, as expected if the former directly regulates the latter (Fig. $4 \mathrm{C}$ ). Consistent with this interpretation, lines carrying the nonreference MIR823A allele and a CMT3 reference allele showed lower CMT3 expression as well as lower $\mathrm{mCHG}$ (Fig. 4B). The specificity of the phenotypic effects and known regulatory mechanism provides strong evidence for a direct causal role for these genes.

Knock-outs of MIR823A in several backgrounds affected CMT3 expression and $\mathrm{MCHG}$ in a manner consistent with this regulatory model (Papareddy et al., 2021), although the effects on methylation were very weak (Fig. 4D, E). The natural polymorphism almost certainly does not involve a loss of function, as there is no common polymorphism in the 21-nt mature miRNA region of MIR823A, nor in the target region of CMT3 (Fig. 4A). There are also no other nonsynonymous polymorphisms in CMT3 significantly associated with the different alleles. 
A

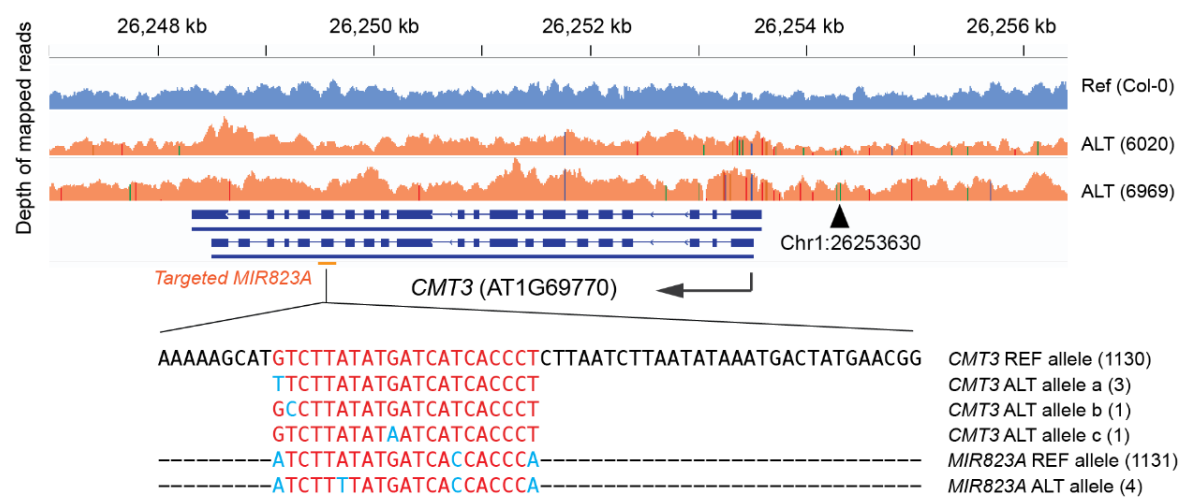

B

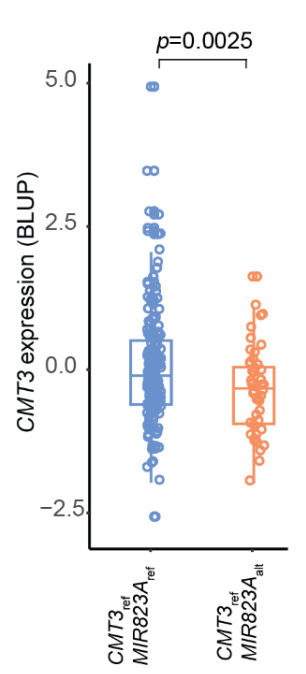

C

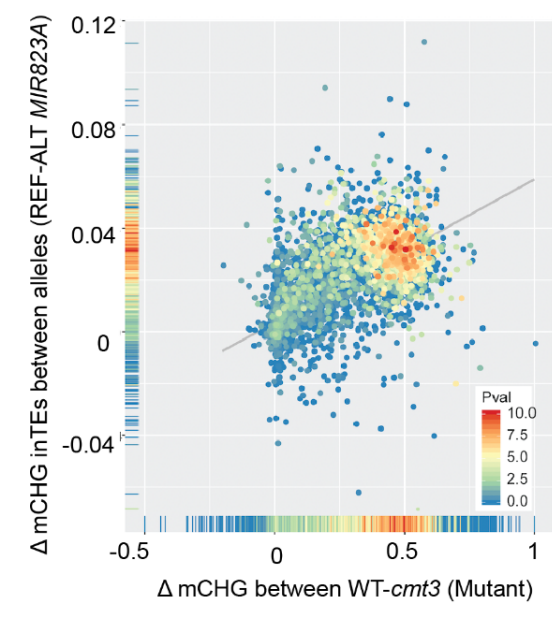

D

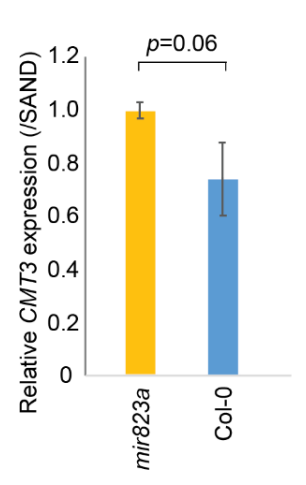

$E$

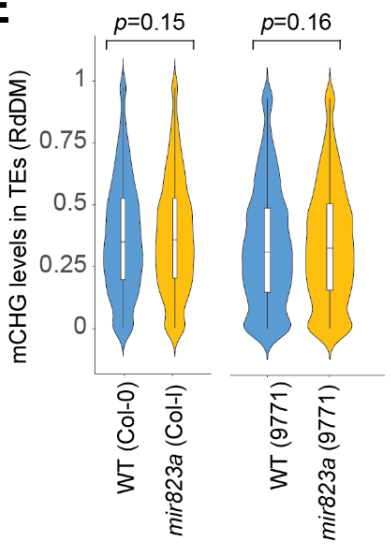

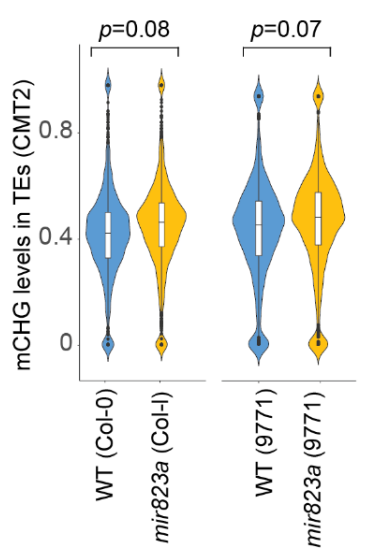

Fig 4. Effects of MIR823A on mCHG levels. (A) Polymorphisms in the miRNA region of MIR823A and the target in CMT3 in 1135 natural lines. Differences from the CMT3 reference sequence are shown in light blue. Haplotype counts are shown in parentheses. (B) Estimated effects of MIR823A alleles on CMT3 expression in natural lines with individual values (Welch's t-test, two-tailed, CMT3 expression was collected from transcriptome data published in Kawakatsu et al., 2016). (C) Scatter plot comparing the average allelic effect of the MIR823A polymorphism with the effect of $c m t 3$ in the Col-0 background. Dots represent individual transposons, and the colors show the significance of the allelic effects as $\log _{10}$-value in GWAS. (D) CMT3 expression in early embryos with standard errors in WT (Col-0) and mir823a. Significance of the difference in mean tested using Welch's t-test ( $n=3$, one-tailed). (E) The distribution of $\mathrm{mCHG}$ levels in RdDM and CMT2-targeted transposons, comparing knock-outs of MIR823A to wildtype in different backgrounds. 


\section{Further evidence for allelic heterogeneity at CMT2}

In addition to de novo establishment of methylation in heterochromatic regions, CMT2 plays a role in $\mathrm{mCHG}$ maintenance through regulation of $\mathrm{H} 3 \mathrm{~K} 9$ methylation via a self-reinforcing loop (Du et al., 2015; Stroud et al., 2014). Previous work has identified two common natural alleles, CMT2a' and CMT2b', affecting mCHH (Dubin et al., 2015; Sasaki et al., 2019; Kawakatsu et al., 2016), but neither appears to affect mCHG (Figs S2, S3, S5). Here we identify a new association, $2 \mathrm{~kb}$ downstream of CMT2 (Fig. 3), that does appear to affect $\mathrm{mCHG}$ (on both RdDM- and CMT2-targeted transposons) (Fig S5). The top SNP is only weakly correlated with CMT2a' and CMT2b' $\left(r^{2}{ }_{C M T 2 b^{\prime}}=0.37 ; r_{C M T 2 a}^{2}=0.24\right)$, but caution is needed when interpreting associations in regions that apparently harbor multiple causal polymorphisms (Sasaki et al., 2021).

\section{A complex association on chromosome 5 includes two a priori genes}

The major peak on chromosome 5 was associated with $\mathrm{mCHG}_{\mid \mathrm{mcHH}}$ on both RdDM- and CMT2-targeted transposons, although the shape of the peak differs slightly. The strongest association was found for CMT2-targeted transposons, and is located 102 bp upstream of a known epigenetic regulator, $M S / 1$, but substantial linkage disequilibrium extends over a $30 \mathrm{~kb}$ region which also includes another a priori gene, ROS3 (Figs 3, S6A).

The loss-of function mutant ros3 does not show altered mCHG in leaves (Fig 1B; Stroud et al., 2013). Loss of MS/1 causes embryonic lethality (Guitton et al., 2004), but the heterozygous mutant msi1-2 does not show a significant effect on $\mathrm{mCHG}$ levels in leaves either. However, MSI1 is required to control DNA methylation via repression of MET1, and a loss of FAS2 in CAF-1 induces mCHG hypermethylation (Fig 1B) (Stroud et al., 2013; Jullien et al., 2008), so MS/1 remains a strong candidate. Furthermore, the strongly associated SNP at Chr5:23555910 could explain almost all phenotypic variation associated with the chromosome 5 peak (Fig S6B), making MSI1 the top candidate, although further experiments are clearly needed.

\section{A jmjC gene is a novel modifier of $\mathrm{mCHG}$ in RdDM-targeted transposons}

The final peak (Chr1:4035022; $-\log _{10} p$-value=10.1) in our study did not pinpoint any a priori gene. However, it is highly significant and narrowly centered on the coding region of a jmjC gene, JUMONJI26 (JMJ26) (Qian et al., 2015). JMJ26 is a close homolog of JMJ25, also known as INCREASE IN BONSAI METHYLATION 1 (IBM1), a histone H3K9m demethylase targeting genic regions in a KYP/SUVH4- and CMT3-dependent manner (Saze et al., 2008; Inagaki et al., 2010). In contrast to IBM1, the function of JMJ26 has barely been studied. Among the most significant associations were two non-synonymous SNPs (Chr1:4035683, 4035690) in the conserved JMJ domain, suggesting allelic effects on the enzymatic activity (Figs 5, S7). 
To explore the function of $J M J 26$, we measured mCHG levels of a loss-of-function mutant, jmj26 (Fig. 5). Unlike IBM1 loss-of-function mutants, jmj26 did not show any morphological phenotype (Fig S8A), but mCHG levels increased significantly in RdDM-targeted transposons (Figs. 5B, C). Furthermore, jmj26 showed increased $\mathrm{mCHG}$ in pericentromeric transposons, whereas ibm1-like hypermethylation was not observed in genic regions (Fig 5D, E). Gene expression was barely affected except for a few DNA methylation sensitive genes, including transposon genes (AT5G35935, AT4G01525) (Table S3). These observations support the functionality of JMJ26 as a histone demethylase with different targets from IBM1, and also makes it a strong a posteriori candidate for causing the GWAS peak on the left end of chromosome 1. 
A

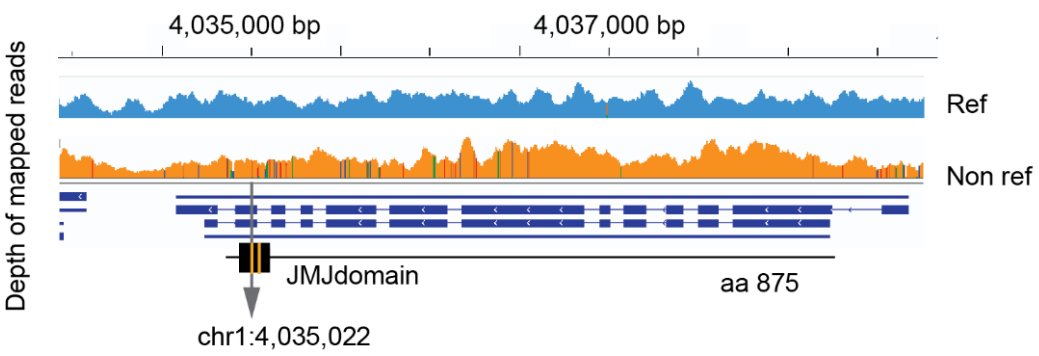

B
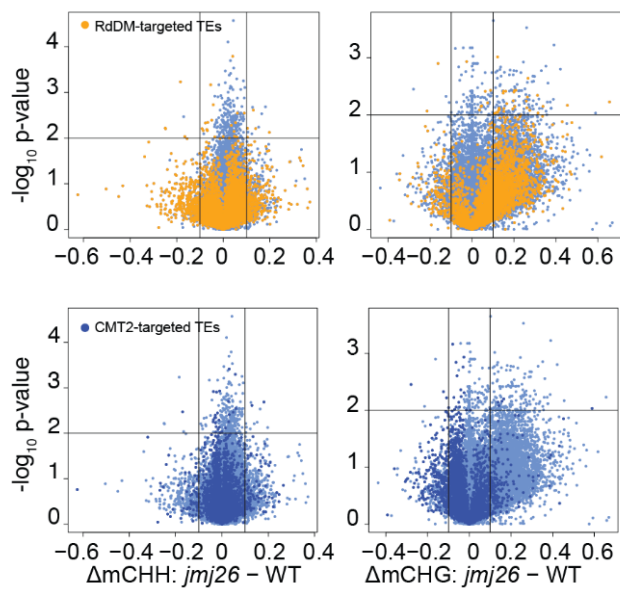

D
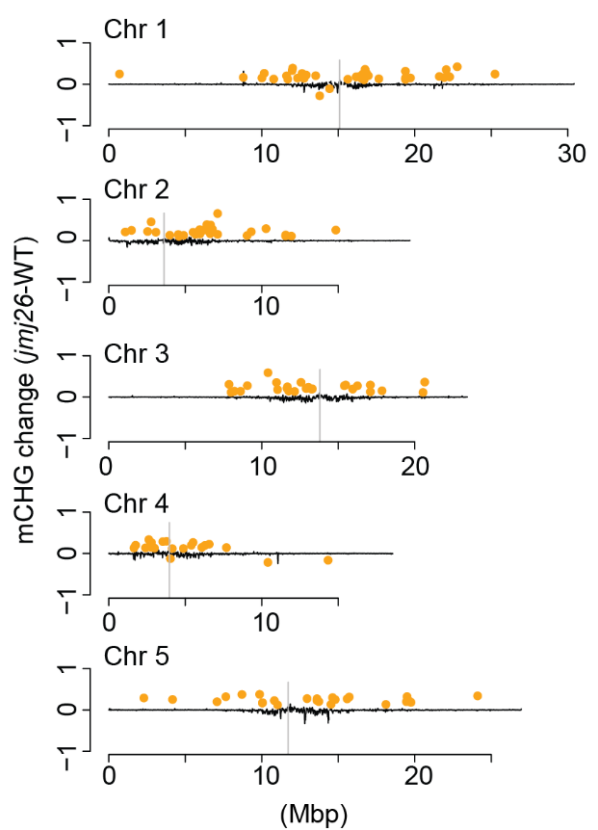

C
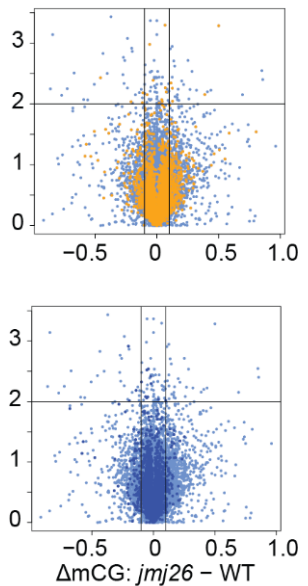

$\mathbf{E}$
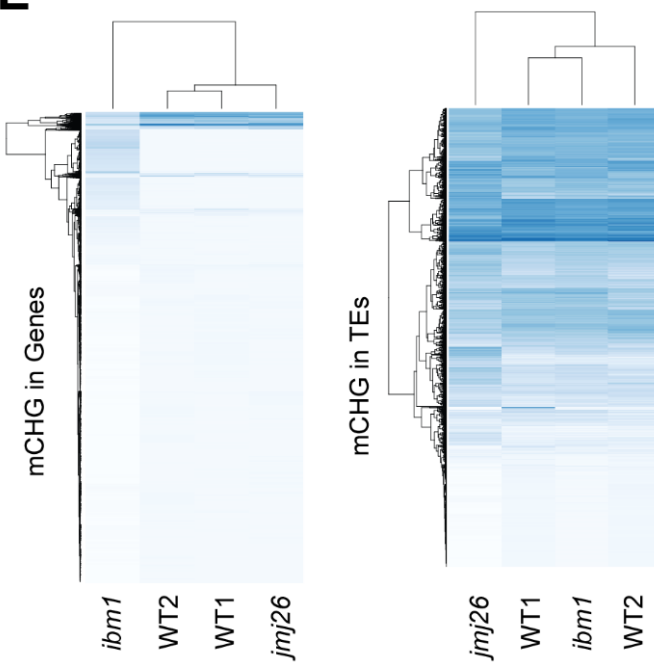

Fig 5. Effects of JMJ26 on mCHG levels. (A) Read density and SNP across the gene model. (B) Volcano plots showing the effects on DNA methylation levels of jmj26 on RdDM- and CMT2-targeted transposons. (C) Violin plots showing distribution of $\mathrm{mCHG}$ levels of RdDM- and CMT2-targeted transposons in WT (Col-0), jmj26, and two lines with JMJ26ref or JMJ26alt transgenes in Col-0 jmj26 background. The gray horizontal line shows the mean value of WT. (D) The genome-wide distribution of transposons affected by JMJ26. Black lines show differential mCHG levels of jmj26 from Col- 0 in 30 $\mathrm{kbp}$ sliding windows. Orange dots show transposons with significantly changed $\mathrm{mCHG}$ levels $(n=2$, $p<0.01$ by two-tailed Welch's $t$-test \& differential mCHG $>0.1$ or $<-0.1$ ). (E) Heat map comparing JMJ26 and IBM1 targets. Rows correspond to standardized $\mathrm{mCHG}$ levels in each gene or transposon for $j \mathrm{mj} 26$, $i b m 1$, and WTs. 


\section{Major loci additively affect $\mathbf{m C H G}$ and influence transposon activity}

Finally, we asked how the five major loci jointly shape $\mathrm{mCHG}$ variation at the population level. Although some of these genetic variants are clearly interacting at the molecular level (e.g., MIR823A directly regulates CMT3), the phenotypic effects appear to be additive (Fig. 6A), and jointly explain a large fraction of the variation: $30.5 \%$ and $14.8 \%$ of $\mathrm{mCHG}_{\mid \mathrm{mCHH}}$ in RdDM- and CMT2-targeted transposons, respectively, while SNP-based heritabilities of these traits are $65 \%$ and $69 \%$. $\mathrm{mCHG}$ levels decrease linearly with the number of $\mathrm{mCHG}$-decreasing alleles, and the single line (out of 774) harboring $\mathrm{mCHG}$-decreasing alleles at all five loci exhibits strong hypo-mCHG ${ }_{\mid \mathrm{mCHH}}$ in RdDM-targeted transposons (Fig. 6A).

These five loci also affect the geographic distribution of $\mathrm{MCHG}$ for both RdDM- and CMT2targeted transposons. In European lines ( $n=971)$, mCHG-decreasing alleles are almost exclusively found in the west, from 0 to $30^{\circ} \mathrm{E}$ (Fig 6B, S9-10). Lines carrying more than three mCHG-decreasing alleles are rare (2.5\%), and the one line carrying all five such alleles is from the Iberian peninsula: IP-Mun-0 (9561), an admixed line of relict and western European descent (1001 Genomes Consortium, 2016).

Interestingly, the longitudinal gradient in the number of $\mathrm{mCHG}$-decreasing alleles is opposite to the longitudinal cline of NRPE1' associated with lower mCHH (Sasaki et al., 2019) and higher transposon mobilization (Baduel et al., 2021) (Fig. 6B). No line was found carrying NRPE1' with more than three $\mathrm{mCHG}$-decreasing alleles, and a permutation test shows that this negative correlation is unlikely to be due to population structure (Fig. 6C, S10C). A plausible alternative is some form of stabilizing selection on methylation levels: multiple $\mathrm{mCHG}$-decreasing alleles in lines with lower $\mathrm{mCHH}$ levels might affect fitness analogously to double knockout mutants of the RdDM and CMT3 pathways that show pleiotropic morphological phenotypes and high transposon transcriptional activity (Cao and Jacobsen, 2002; Stroud et al., 2014; Chan et al., 2006).

To explore this further, we examined the effect of $\mathrm{mCHG}$-negative alleles on transposon mobilization by intersecting our data with those of Baduel et al. (2021), who found a significant correlation between recent transposon mobilization and the NRPE1' non-reference allele. We found that this effect reflects an epistatic interaction with the five loci reported here: significantly increased transposon mobilization is only found in lines carrying the NRPE1' nonreference allele and two or more $\mathrm{mCHG}$-decreasing alleles ( $p$-value $<0.01$; Figs $6 \mathrm{D}, \mathrm{S} 11$ ). In particular, lines carrying three mCHG-decreasing alleles and the NRPE1' non-reference allele have about thirty times more recent transposon insertions than lines carrying no $\mathrm{mCHG}$ decreasing alleles and the NRPE1' non-reference allele. Such lines are not closely related and carry different transposon insertions, mostly Copia and MuDR. They are found in two distinct regions: north-western Africa, including the Canary Islands, and the south-eastern Balkans (Fig. 6B, Table S4). 
A

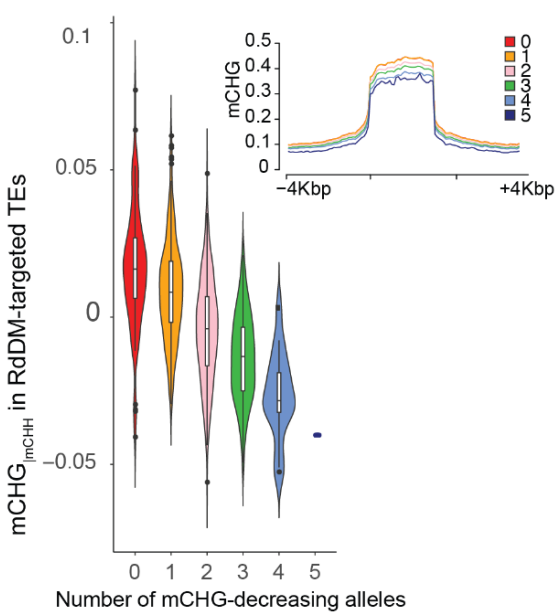

B
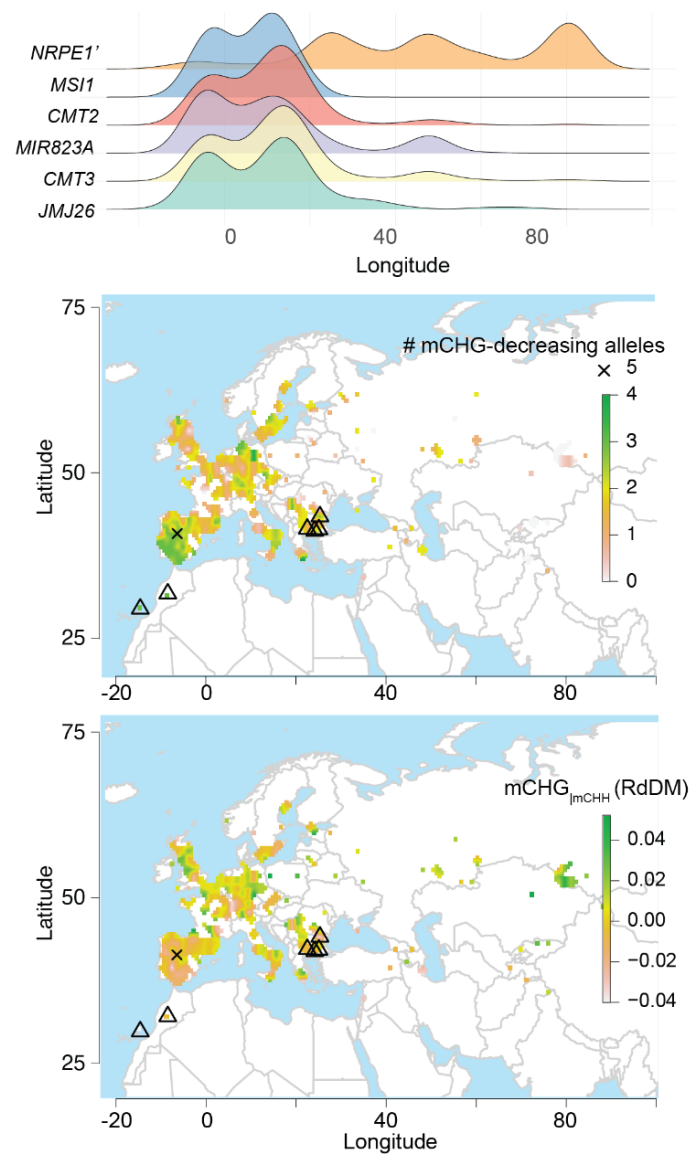

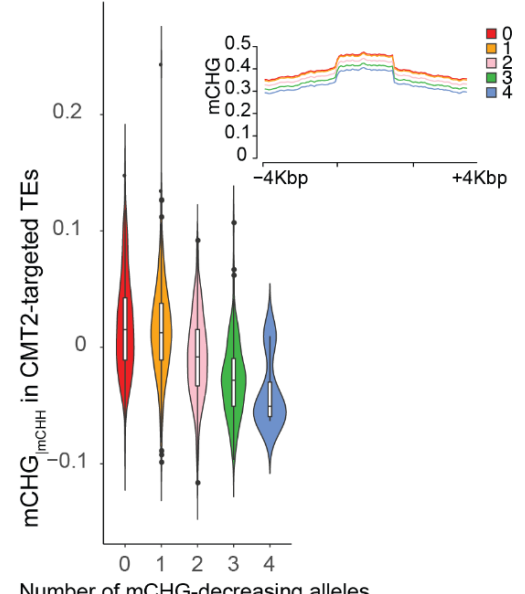

C

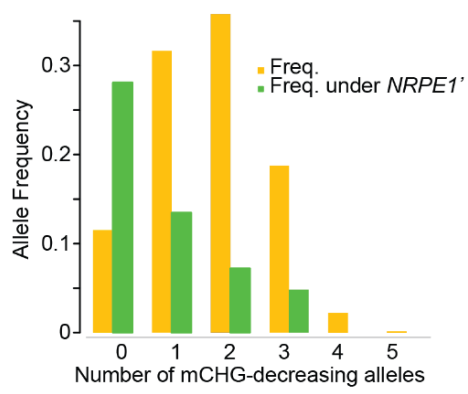

D

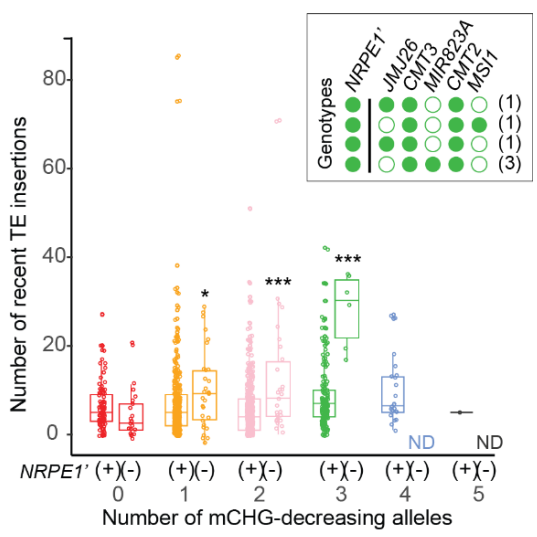

Fig 6. Cumulative effects and geographic distribution of mCHG-decreasing alleles. (A) Cumulative effect on $\mathrm{mCHG}_{\mid \mathrm{mCHH}}$. Chr1:4035022 (JMJ26) was excluded from the analysis of CMT2targeted transposons as it has no effect. Chr5:2355910 was used for MSI1. Inserted plots show mCHG levels around transposons, calculated using 20 sliding windows across the transposon body and flanking regions. (B) Longitudinal frequency distribution of $\mathrm{mCHG}$-decreasing and NRPE1' alleles (top); geographical distribution of the number of $\mathrm{mCHG}$-decreasing alleles (middle), and $\mathrm{mCHG}_{\mid \mathrm{mCH}}$ levels in RdDM-targeted transposons (bottom). Triangles correspond to lines in the inserted plot of panel D. (C) The frequency of distribution of the number of $\mathrm{mCHG}$-decreasing alleles, separately for NRPE1' genotypes. (D) The number of recent transposon insertions (Baduel et al., 2021) as a function of NRPE1' genotype and the number of $\mathrm{mCHG}$-decreasing alleles. Significance was tested using a permutation test (see methods; ${ }^{* * *}$ is $p<0.01,{ }^{*}$ is $p<0.1$ ). The insert shows the six observed genotypes with three $\mathrm{mCHG}$-decreasing and the NRPE1' non-reference allele. Filled circles indicate $\mathrm{mCHH}-$ or mCHG-decreasing alleles. 


\section{Discussion}

\section{The genetics of non-CG transposon methylation}

Non-CG methylation in plants is associated with gene silencing, especially for transposons. In previous studies, we identified an oligogenic architecture for $\mathrm{mCHH}$ on transposons involving major polymorphism at CMT2, NRPE1, AGO1, and AGO9 - all involved in DNA methylation pathways (Kawakatsu et al., 2016; Dubin et al., 2015; Sasaki et al., 2019). Here we complement this with a conditional GWAS approach and identify five major polymorphisms affecting $\mathrm{mCHG}$, presumably acting via methylation maintenance pathways: CMT2 (different alleles), CMT3, MIR823A (directly regulating CMT3), MSI1 (probably), and JMJ26. All had previously been shown to affect DNA methylation except JMJ26.

We observed major polymorphisms at several of the layers of regulation controlling mCHG, including methylation, de-methylation, and histone modification - even posttranscriptional regulation, in the form of a microRNA targeting the CMT3 pathway directly. The $D D M 1$ and $F A S 1$ alleles detected using non-stringent threshold would further contribute to the fine-tuning of DNA methylation over transposons genome-wide.

The genetic architecture revealed by these studies is very different from the "omnigenic" model (Boyle et al., 2017; Liu et al., 2019) which has come to represent a typical human GWAS result. It is also different from trans-regulation for reported human mCG variation (Hawe et al., 2022; Villicaña and Bell, 2021). Rather than a diffuse genetic architecture involving huge numbers of loci of small effect and unknown causal relationship to the phenotype, we find a small number of major loci with highly plausible mechanistic connection to the phenotype - and a diffuse background for which we lack power to dissect further. The results are reminiscent of those found for other clinal traits, like flowering time (Yeaman et al., 2016; Atwell et al., 2010), flower color (Ortiz-Barrientos, 2013), or eye and coat color in mammals (Miller et al., 2007; Beleza et al., 2013; Lloyd-Jones et al., 2017), suggesting that transposon methylation is likewise under selection, most likely as part of a plastic and environmentally sensitive genome defense system - a notion further supported by the association with transposon mobility reported here and be Baduel et al. (2021).

\section{The power and complexity of conditional GWAS}

The performance of GWAS relies on using the right model for the relation between genotype and phenotype. As with other statistical methods, using the wrong model may lead to unpredictable results. However, lack of prior knowledge makes modeling difficult, and univariate linear models are therefore the default in GWAS. Here we focus on correlated traits. Correlations between traits may arise for a variety of reasons, mostly obviously a shared genetic basis (pleiotropy), and it is clear that such traits should, in principle, be analyzed jointly using some kind of multivariate analysis (Califano et al., 2012; Korte et al., 2012; Stegle et al., 
2012; Stephens, 2013). Our results provide a dramatic illustration of the potential benefits of doing so. Despite high heritability, univariate GWAS of $\mathrm{mCHG}$ variation failed to detect any significant associations, leading us to conclude, erroneously, that the trait was simply too polygenic (Kawakatsu et al., 2016). In contrast, GWAS of the same data using a conditional model that controlled for $\mathrm{mCHH}$ revealed an oligogenic architecture with five major loci qualitatively similar to what we had previously seen for $\mathrm{mCHH}$ using univariate GWAS, also in that it mainly identified loci corresponding to biologically meaningful a priori candidates (Kawakatsu et al., 2016; Dubin et al., 2015; Sasaki et al., 2019).

However, while our conditional GWAS approach clearly improved power to reject the null hypothesis of no association, interpreting the result in terms of causality is more difficult. We believe that, by controlling for $\mathrm{mCHH}$, we have effectively simplified the trait, revealing genetic factors affecting $\mathrm{mCHG}$ only, perhaps by affecting the maintenance of this type of DNA methylation. There is considerable background experimental evidence to support such a model. For example, the RdDM pathway would affect both $\mathrm{mCHH}$ and $\mathrm{mCHG}$ via de novo methylation, while $\mathrm{mCHG}$ (but not $\mathrm{mCHH}$ ) would be also maintained by the CMT3 pathway, and by controlling the former, we would reveal the latter.

Our GWAS results are consistent with this interpretation. Polymorphism at NRPE1, a key component of the RdDM pathway, was revealed in univariate GWAS, while variation in CMT3 (and its regulator, MIR823a) was only found after controlling for $\mathrm{mCHH}$. At the same time, it seems clear that reality is more complex, and that both $\mathrm{mCHH}$ and $\mathrm{mCHG}$ are regulated by multiple homeostatic mechanisms that also involve factors not included in our model, like histone modifications (Zhang et al., 2021). It is therefore not surprising that most of the polymorphisms we have identified seem to affect both traits, albeit not to the same extent (exceptions include the one at MIR823A, which only seems to affect $\mathrm{mCHG}$, and the previously identified CMT2a' and CMT2b' polymorphisms, which only affect $\mathrm{mCHH}$ on CMT2targeted transposons; see Figs S2, S3).

Two other GWAS models produced consistent results, but had less power (Fig S12, S13). A fully parameterized multi-trait mixed model for $\mathrm{mCHH}$ and $\mathrm{mCHG}$ (MTMM; see Korte et al., 2012) confirmed that the new associations presented in this paper were "specific" to one of the two traits, while a conditional model for $\mathrm{mCHH}$ while controlling for $\mathrm{mCHG}\left(\mathrm{mCHH}_{\mid \mathrm{mCHG}}\right)$ also identified most of these loci, plus the previously identified CMT2 associations (Dubin et al., 2015; Sasaki et al., 2019), which affect mCHH only.

In conclusion, we agree with Stephens (2013) that multi-trait association methods can have much greater power than univariate methods, but require an appropriate statistical framework for interpretation. However, in a model organism like $A$. thaliana, further experiments should guide the construction of such a framework. 


\section{Functional importance of non-CG transposon methylation}

While genomics has revealed striking geographic variation in DNA methylation, and an equally striking genetic architecture underlying this, the functional importance of all this variation has been rather less clear. Based on decades of molecular biology studies, it is reasonable to speculate that it must play some role in regulating transposon activity (Stroud et al., 2013; Kim and Zilberman, 2014; Pikaard and Mittelsten Scheid, 2014; Matzke et al., 2015). Non-CG methylation is part of a redundant system with strong epistasis between $\mathrm{mCHH}$ and $\mathrm{mCHG}$ in keeping transposons transcriptionally silent (Cao and Jacobsen, 2002; Stroud et al., 2014; Chan et al., 2006). However, direct evidence based on transposon mobility in nature has been missing. Now, the evidence is gathering that the allelic variants responsible for transposon methylation variation do indeed affect transposon mobility. Baduel et al (2021) showed that the NRPE1' polymorphism identified as affecting $\mathrm{mCHH}$ on RdDM-targeted transposons by Sasaki et al (2019) is associated with recent transposon mobility, and we show here that the multiple loci controlling $\mathrm{mCHG}$ appear to play a similar role. Our findings connect complex genome defense systems proposed in molecular biology studies with natural populations. Furthermore, the geographic distribution of the relevant multi-locus genotypes suggests that selection may be acting to maintain the appropriate level of transposon silencing - for unknown reasons. Further studies, such as crosses with new allele combinations and common garden experiments, will be required to address these questions.

\section{Materials and methods}

\section{Analyzed data sets}

\section{DNA methylation data}

The DNA methylation data sets are summarized in Table S5. Briefly, we analyzed a bisulfitesequencing data set published in the 1001 epigenome project (Kawakatsu et al., 2016), 85 epigenetic-related mutants (Stroud et al., 2013), and our own sequence data described below. All reads were mapped on the appropriate pseudo-genome provided by the 1001 genome project (1001 Genomes Consortium., 2016) using a Methylpy pipeline v1.2 (Schultz et al., 2015). DNA methylation levels were estimated as weighted methylation levels for each transposon defined in Araport11 annotation. CMT2- and RdDM-targeted transposons were defined as having differential levels of methylation ( $>0.1)$ between wild-type and cmt2 or drm1drm2 in Col-0 previously described in Kawakatsu et al. (2016). For each line, average DNA methylation was calculated using all transposons for which at least one read was mapped. 


\section{RNA-seq data}

The RNA-seq data are summarized in Table S5. Quality control and adapter trimming of all RNA-seq data were conducted using FASTP (Chen et al., 2018). Adapter trimmed reads were aligned on the reference genome (Araport11; Cheng et al., 2017) using STAR v2.7 (Dobin et al., 2013) with default settings. For all annotated genes, mapped read counts were calculated using featureCounts v2.0.1 in the Subread package (Liao et al., 2013), accepting reads that were mapped on multiple genes (option $-\mathrm{M}$ ). Using the count data, differentially expressed genes were detected by edgeR v3.1 (Robinson et al., 2010) with glmQLFTest() function (FDR $<0.05)$. For transposon expression, reads were mapped as described above but allowing multiple hits (STAR --outFilterMultimapNmax 100 and --winAnchorMultimapNmax 100). Mapped reads were calculated by TEtoolkits (Jin et al., 2015) using the TEcount() function, and differentially expressed transposons were detected using edgeR. CMT3 expression in natural populations were downloaded from the 1001 epigenome project data (Kawakatsu et al., 2016).

\section{Statistics}

\section{Genome-wide association studies}

Univariate and conditional GWAS were carried out using LIMIX (Lippert et al., 2014) version 3.0.4 with a full genome SNP matrix for 774 lines (Kawakatsu et al., 2016) from the 1001 genome project $(10,709,949$ SNPs) and the following linear mixed model (LMM),

$$
\begin{aligned}
& Y=\alpha L+\beta X+g+e \\
& \operatorname{var}(Y)=\sigma_{g}^{2} K+\sigma_{g}^{2} I
\end{aligned}
$$

where $Y$ is the $n \times 1$ vector of a phenotype, fixed terms, $L$ and $X$, are the $n \times 1$ vectors corresponding to a covariate and a genotype to be tested (SNP) with the parameters $\alpha$ and $\beta$, respectively. $g \sim \mathcal{N}\left(0, \sigma_{g}^{2} K\right)$ and $e \sim \mathcal{N}\left(0, \sigma_{e}^{2} I\right)$ are random terms, including the identity-bystate kinship matrix $K$ representing the genetic relatedness (Yu et al., 2006; Kang et al., 2008) and the residuals, respectively. $Y, L$, and $X$ were z-scored. Univariate models without cofactor take $\alpha=0$. SNPs that satisfied minor allele frequency $>5 \%$ were used for association studies. Bonferroni-correction was used for multiple-testing correction after excluding all SNPs less than MAF 5\%. Multi-trait linear mixed models (Fig. S12) were performed to identify common genetic effects (common) and differing genetic effects (specific) between two traits using LIMIX (Lippert et al., 2014) following models described in Korte et al. (2012).

\section{Enrichment test}

To assess GWAS results, we calculated FDR and enrichment of a priori gene for each GWAS result (as described in Atwell et al. (2010)) using a list of 79 epigenetic regulators from 
Kawakatsu et al. (2016). The most significant $p$-value within $15 \mathrm{~kb}$ of a gene (MAF $>5 \%$ ) was assigned as the significance of the gene.

\section{Heritability}

SNP-heritability was estimated using REML implemented in mixmogam (https://github.com/bvilhjal/mixmogam/blob/master/phenotypeData.py). The proportion of phenotypic variation explained by identified genetic variants was calculated using $r^{2}$ (Nakagawa and Schielzeth, 2013) using R scripts published in Sasaki et al. (2018).

\section{Correlation of the allelic effects and molecular phenotypes}

For 9329 transposons common across 774 lines, differential mCHG levels induced by alleles were estimated for each transposon as the differential average methylation level between lines carrying the reference ( $\mathrm{Col}-0)$ and the alternative allele. Differential $\mathrm{mCHG}$ levels induced by mutants were calculated analogously (Stroud et al., 2013). The details are described in Sasaki et al. (2019).

\section{Gene effect of loss-of-function mutants}

Gene functions of MIR823A and JMJ26 were tested using transgenic lines described below. We measured DNA methylation levels for two or three independently grown plants, and the significance of differential mean values across RdDM- and CMT2-targeted transposons was tested by Welch's t-test (two-tailed). DNA methylation pattern of jmj26 was compared to ibm1 and the control samples from published data sets (Stroud et al., 2013).

\section{Permutation tests}

We evaluated effects of $\mathrm{mCHG}-$ decreasing alleles using permutation tests with 3000 randomly chosen SNP-sets with the same allele frequencies as the identified alleles along the genome. For correlations between number of $\mathrm{mCHG}$-decreasing alleles and longitude or linkage disequilibrium between the five alleles and NRPE1' alleles, we compared the observed to the 3000 randomly chosen samples directly. For evaluation of epistatic effects between NRPE1' and $\mathrm{mCHG}$-decreasing alleles on transposon activity, we randomly chose 3000 SNPs with the same allele frequency as NRPE1' and tested the effect of zero to five $\mathrm{mCHG}$-decreasing alleles.

\section{Plant materials}

Plants were grown at the $21^{\circ} \mathrm{C}$ with a 16 -h light/8-h dark cycle and humidity $60 \%$. Whole plant rosettes were harvested individually when they reached the 9-true-leaf stage of development. The t-DNA insertion line of jmj26 (SALKseq_069498.1) and a gamma-ray mutant msi1-2 (Jullien et al., 2008) were purchased from Nottingham Arabidopsis Stock Center (http://arabidopsis.info/) for the functional analysis. Homozygous jmj26 was used for bisulfite sequencing after the second generation. msi1-2 was maintained as a segregating population, 
and heterozygous lines were selected by genotyping and measuring MIS1 expression by qRTPCR.

\section{Transgenic lines}

\section{CRISPR/CAS9 mutant lines}

Loss-of-function mutants mir823a for Col-0 and 9771 were generated using CRISPR/CAS9 methods described in Xing et al (2014). Briefly, to generate a vector carrying two sgRNAs targeting MIR823A, the target sites were incorporated into forward and reverse PCR primers, and the fragment amplified from pCBC-DT1T2 (Table S6). Subsequently, an amplified and purified fragment was assembled using the Golden Gate reaction into pHSE401E modified to contain a mCherry marker. The resulting vector was transformed into Agrobacterium tumefaciens GV3101/pSOUP, then transformed into $A$. thaliana by the floral dip method (Zhang et al., 2006). T1 seeds were screened by mCherry marker under the fluorescence stereomicroscope (Discovery.V8, Zeiss), and the mutations were genotyped by Sanger sequencing. T2 seeds without mCherry signal were kept to amplify the stable T3 generations, which were used for further analyses.

\section{Rescue analyses}

The gene function of JMJ26 was confirmed by rescue experiments. The protein-coding genomic sequence including $3 \mathrm{kbp}$ promoter sequence was PCR-amplified and cloned into the pGreen0029 binary vector (Hellens et al., 2000) using In-Fusion Cloning system (Takara Bio Europe, Saint-Germain-en-Laye, France) for Col-0 and DralV 6-22 (5984) carrying the reference and the alternative allele, respectively. These constructs were used for floral dipping transformation to Col-0 background jmj26 (SALKseq_069498.1) to create transgenic lines (Zhang et al., 2006).

\section{mRNA abundance}

Total RNA was extracted using ZR Plant MiniPrep Kit (Zymo Research) including a DNase I treatment and was quantified by fluorometer Qubit 4 (Invitrogen). cDNA was synthesized using the SuperScript III First-Strand Synthesis System (Invitrogen) according to the manufacturer's protocols. qRT-PCR was performed using the LightCycler 96 system (Roche) with FastStart Essential DNA Green Master (Roche). The transcript abundance was estimated by ddCT methods with an internal control SAND (AT2G28390) (Czechowski et al., 2005). Primer information is listed in Table S6. 


\section{MicroRNA abundance}

\section{Material collection and RNA extraction}

Siliques were collected five days after pollination when the embryos reached an early torpedo stage according to the previous report (Papareddy et al., 2021). Using the tungsten needles, seeds were dissected from siliques and collected in $1.5 \mathrm{ml}$ tubes, and washed 3-4 times with fresh 10\% RNA later solution (Thermo Fisher Scientific). After the last wash, the buffer was replaced with $500 \mu \mathrm{ITRIzol}{ }^{\mathrm{TM}}$ Reagent (Ambion) and total RNA was isolated as described in Hofmann et al. (2019).

\section{qRT-PCR Analysis}

The quantification was conducted according to the protocol in Papareddy et al. (2021). Briefly. cDNA synthesis was performed using SuperScript III System (Invitrogen), and the corresponding stem-loop primers were added to the reverse transcription reaction. qRT-PCR analysis was performed on the LightCycler 96 Instrument (Roche) with the FastStart Essential DNA Green Master (Roche). The transcript abundance was estimated by ddCT methods with an internal control miRNA160.

\section{RNA sequencing}

RNA was extracted in the same protocol with qRT-PCR for mRNA.Total RNA was treated with the poly(A) RNA Selection Kit (Lexogen). Libraries were prepared according to the manufacturer's protocol in NEBNext Ultra II Directional RNA Library Prep Kit (New England BioLabs) and individually indexed with NEBNext Multiplex Oligos for Illumina (New England BioLabs). The quantity and quality of each amplified library were analyzed by using Fragment Analyzer (Agilent) and HS NGS Fragment Kit (Agilent).

\section{Bisulfite sequencing}

Genomic DNA was extracted using GeneJET Plant Genomic DNA Purification Kit (Thermo Scientific) and sheared by E220 Focused-ultrasonicator (Covaris) to target average fragments around 350 bp. Sequencing libraries were prepared using NebNext Ultra II DNA Library Prep Kit (New England BioLabs) with Methylated Adaptor (New England BioLabs). Adapter-ligated DNA was carried through the bisulfite conversion using EZ-96 DNA Methylation-Gold ${ }^{\mathrm{TM}}$ MagPrep Kit (Zymo Research). Bisulfite-treated samples were amplified by EpiMark Hot Start Taq DNA Polymerase and indexed with NEBNext Multiplex Oligos for Illumina (New England BioLabs). All libraries were sequenced by Illumina NextSeq550 or Hiseq2500.

\section{Data availability}

All data are summarized in Table S5. Sequencing data have been deposited to GSE194406. 


\section{Acknowledgments}

We are grateful to Fred Berger, Tetsuji Kakutani, Arturo Marí-Ordóñez, Ortrun Mittelsten Scheid and all member of the Nordborg lab for discussions, and to Pierre Baduel, Vincent Colot, and Leandro Quadrana for discussions and sharing of transposon mobilization data. We thank Pierre Baduel, Pieter Clauw, Vincent Colot, Thomas Ellis, and Bob Schmitz for extremely helpful comments on the manuscript, Jian-Kang Zhu and Kai Tang for sharing ros3 seeds and methyl-C seq data, and Rahul Pisupati for help with data analyses. The bisulfite and RNA sequencing were performed by the Next Generation Sequencing Facility at Vienna BioCenter Core Facilities (VBCF), a member of the Vienna BioCenter (VBC), Austria. This work was funded in part by ERC AdvG 789037 EPICLINES to MN.

\section{Conflict of Interest}

The authors have no conflict of interest to declare.

\section{Supplemental tables}

Table S1. Linkage disequilibrium between five loci

Table S2. a priori genes identified by enrichment tests (FDR=0.2)

Table S3. The list of genes significantly affected in jmj26

Table S4. Lines carrying three $\mathrm{mCHG}$-decreasing alleles with $\mathrm{mCHH}$-decreasing NRPE1' allele

Table S5. Key resources

Table S6. Primer list

\section{References}

1001 Genomes Consortium (2016). 1,135 Genomes Reveal the Global Pattern of Polymorphism in Arabidopsis thaliana. Cell 166: 481-491.

Atwell, S. et al. (2010). Genome-wide association study of 107 phenotypes in Arabidopsis thaliana inbred lines. Nature 465: 627-631.

Baduel, P. and Colot, V. (2021). The epiallelic potential of transposable elements and its evolutionary significance in plants. Philos. Trans. R. Soc. Lond. B Biol. Sci. 376: 20200123.

Baduel, P., Leduque, B., Ignace, A., Gy, I., Gil, J., Loudet, O., Colot, V., and Quadrana, L. (2021). Genetic and environmental modulation of transposition shapes the evolutionary potential of Arabidopsis thaliana. Genome Biol. 22: 1-26. 
Becker, C., Hagmann, J., Müller, J., Koenig, D., Stegle, O., Borgwardt, K., and Weigel, D. (2011). Spontaneous epigenetic variation in the Arabidopsis thaliana methylome. Nature 480: 245-249.

Beleza, S. et al. (2013). Genetic architecture of skin and eye color in an African-European admixed population. PLoS Genet. 9: e1003372.

Boyle, E.A., Li, Y.I., and Pritchard, J.K. (2017). An Expanded View of Complex Traits: From Polygenic to Omnigenic. Cell 169: 1177-1186.

Califano, A., Butte, A.J., Friend, S., Ideker, T., and Schadt, E. (2012). Leveraging models of cell regulation and GWAS data in integrative network-based association studies. Nat. Genet. 44: 841-847.

Cao, X. and Jacobsen, S.E. (2002). Locus-specific control of asymmetric and CpNpG methylation by the DRM and CMT3 methyltransferase genes. Proc. Natl. Acad. Sci. U. S. A. 99 Suppl 4: 16491-16498.

Chan, S.W.-L., Henderson, I.R., Zhang, X., Shah, G., Chien, J.S.-C., and Jacobsen, S.E. (2006). RNAi, DRD1, and histone methylation actively target developmentally important non-CG DNA methylation in arabidopsis. PLoS Genet. 2: e83.

Cheng, C.-Y., Krishnakumar, V., Chan, A.P., Thibaud-Nissen, F., Schobel, S., and Town, C.D. (2017). Araport11: a complete reannotation of the Arabidopsis thaliana reference genome. Plant J. 89: 789-804.

Chen, S., Zhou, Y., Chen, Y., and Gu, J. (2018). fastp: an ultra-fast all-in-one FASTQ preprocessor. Bioinformatics 34: i884-i890.

Chuong, E.B., Elde, N.C., and Feschotte, C. (2017). Regulatory activities of transposable elements: from conflicts to benefits. Nat. Rev. Genet. 18: 71-86.

Czechowski, T., Stitt, M., Altmann, T., Udvardi, M.K., and Scheible, W.-R. (2005). Genome-Wide Identification and Testing of Superior Reference Genes for Transcript Normalization in Arabidopsis. Plant Physiol. 139: 5-17.

Deniz, Ö., Frost, J.M., and Branco, M.R. (2019). Regulation of transposable elements by DNA modifications. Nat. Rev. Genet. 20: 417-431.

Dobin, A., Davis, C.A., Schlesinger, F., Drenkow, J., Zaleski, C., Jha, S., Batut, P., Chaisson, M., and Gingeras, T.R. (2013). STAR: ultrafast universal RNA-seq aligner. Bioinformatics 29: 15-21.

Dubin, M.J. et al. (2015). DNA methylation in Arabidopsis has a genetic basis and shows evidence of local adaptation. Elife 4: e05255.

Du, J., Johnson, L.M., Jacobsen, S.E., and Patel, D.J. (2015). DNA methylation pathways and their crosstalk with histone methylation. Nat. Rev. Mol. Cell Biol. 16: 519-532.

East, E.M. (1910). A Mendelian Interpretation of Variation that is Apparently Continuous. Am. Nat. 44: 65-82.

Feng, S., Zhong, Z., Wang, M., and Jacobsen, S.E. (2020). Efficient and accurate determination of genome-wide DNA methylation patterns in Arabidopsis thaliana with enzymatic methyl sequencing. Epigenetics Chromatin 13: 42.

Gallagher, M.D. and Chen-Plotkin, A.S. (2018). The Post-GWAS Era: From Association to Function. Am. J. Hum. Genet. 102: 717-730. 
Goll, M.G., Kirpekar, F., Maggert, K.A., Yoder, J.A., Hsieh, C.-L., Zhang, X., Golic, K.G., Jacobsen, S.E., and Bestor, T.H. (2006). Methylation of tRNAAsp by the DNA methyltransferase homolog Dnmt2. Science 311: 395-398.

Guitton, A.-E., Page, D.R., Chambrier, P., Lionnet, C., Faure, J.-E., Grossniklaus, U., and Berger, F. (2004). Identification of new members of Fertilisation Independent Seed Polycomb Group pathway involved in the control of seed development in Arabidopsis thaliana. Development 131: 2971-2981.

Gutzat, R. et al. (2020). Arabidopsis shoot stem cells display dynamic transcription and DNA methylation patterns. EMBO J. 39: e103667.

Hawe, J.S. et al. (2022). Genetic variation influencing DNA methylation provides insights into molecular mechanisms regulating genomic function. Nat. Genet.

Hellens, R., Mullineaux, P., and Klee, H. (2000). Technical Focus:A guide to Agrobacterium binary Ti vectors. Trends in Plant Science 5: 446-451.

Hennig, L., Bouveret, R., and Gruissem, W. (2005). MSI1-like proteins: an escort service for chromatin assembly and remodeling complexes. Trends Cell Biol. 15: 295-302.

Hennig, L., Taranto, P., Walser, M., Schönrock, N., and Gruissem, W. (2003). Arabidopsis MSI1 is required for epigenetic maintenance of reproductive development. Development 130: 2555-2565.

Hofmann, F., Schon, M.A., and Nodine, M.D. (2019). The embryonic transcriptome of Arabidopsis thaliana. Plant Reprod. 32: 77-91.

Hüther, P., Hagmann, J., Nunn, A., Kakoulidou, I., Pisupati, R., Langenberger, D., Weigel, D., Johannes, F., Schultheiss, S.J., and Becker, C. (2022). MethylScore, a pipeline for accurate and context-aware identification of differentially methylated regions from population-scale plant WGBS data. bioRxiv: 2022.01.06.475031.

Inagaki, S., Miura-Kamio, A., Nakamura, Y., Lu, F., Cui, X., Cao, X., Kimura, H., Saze, H., and Kakutani, T. (2010). Autocatalytic differentiation of epigenetic modifications within the Arabidopsis genome. EMBO J. 29: 3496-3506.

Jackson, J.P., Lindroth, A.M., Cao, X., and Jacobsen, S.E. (2002). Control of CpNpG DNA methylation by the KRYPTONITE histone H3 methyltransferase. Nature 416: 556 560.

Jin, Y., Tam, O.H., Paniagua, E., and Hammell, M. (2015). TEtranscripts: a package for including transposable elements in differential expression analysis of RNA-seq datasets. Bioinformatics 31: 3593-3599.

Johannes, F. and Schmitz, R.J. (2019). Spontaneous epimutations in plants. New Phytol. 221: 1253-1259.

Jullien, P.E., Mosquna, A., Ingouff, M., Sakata, T., Ohad, N., and Berger, F. (2008). Retinoblastoma and its binding partner MSI1 control imprinting in Arabidopsis. PLoS Biol. 6: e194.

Kang, H.M., Zaitlen, N.A., Wade, C.M., Kirby, A., Heckerman, D., Daly, M.J., and Eskin, E. (2008). Efficient control of population structure in model organism association mapping. Genetics 178: 1709-1723.

Kawakatsu, T. et al. (2016). Epigenomic Diversity in a Global Collection of Arabidopsis thaliana Accessions. Cell 166: 492-505. 
Kawashima, T. and Berger, F. (2014). Epigenetic reprogramming in plant sexual reproduction. Nat. Rev. Genet. 15: 613-624.

Kim, M.Y. and Zilberman, D. (2014). DNA methylation as a system of plant genomic immunity. Trends Plant Sci. 19: 320-326.

Korte, A., Vilhjálmsson, B.J., Segura, V., Platt, A., Long, Q., and Nordborg, M. (2012). A mixed-model approach for genome-wide association studies of correlated traits in structured populations. Nature Genetics 44: 1066-1071.

Lande, R. and Arnold, S.J. (1983). The measurement of selection on correlated characters. Evolution 37: 1210-1226.

Law, J.A. and Jacobsen, S.E. (2010). Establishing, maintaining and modifying DNA methylation patterns in plants and animals. Nat. Rev. Genet. 11: 204-220.

Liao, Y., Smyth, G.K., and Shi, W. (2013). The Subread aligner: fast, accurate and scalable read mapping by seed-and-vote. Nucleic Acids Res. 41: e108.

Lindroth, A.M., Cao, X., Jackson, J.P., Zilberman, D., McCallum, C.M., Henikoff, S., and Jacobsen, S.E. (2001). Requirement of CHROMOMETHYLASE3 for maintenance of CpXpG methylation. Science 292: 2077-2080.

Lippert, C., Casale, F.P., Rakitsch, B., and Stegle, O. (2014). LIMIX: genetic analysis of multiple traits. bioRxiv.

Liu, X., Li, Y.I., and Pritchard, J.K. (2019). Trans Effects on Gene Expression Can Drive Omnigenic Inheritance. Cell 177: 1022-1034.e6.

Lloyd-Jones, L.R., Robinson, M.R., Moser, G., Zeng, J., Beleza, S., Barsh, G.S., Tang, H., and Visscher, P.M. (2017). Inference on the Genetic Basis of Eye and Skin Color in an Admixed Population via Bayesian Linear Mixed Models. Genetics 206: 1113-1126.

Matzke, M.A., Kanno, T., and Matzke, A.J.M. (2015). RNA-Directed DNA Methylation: The Evolution of a Complex Epigenetic Pathway in Flowering Plants. Annu. Rev. Plant Biol. 66: 243-267.

Meng, D., Dubin, M., Zhang, P., Osborne, E.J., Stegle, O., Clark, R.M., and Nordborg, M. (2016). Limited Contribution of DNA Methylation Variation to Expression Regulation in Arabidopsis thaliana. PLoS Genet. 12: e1006141.

Miller, C.T., Beleza, S., Pollen, A.A., Schluter, D., Kittles, R.A., Shriver, M.D., and Kingsley, D.M. (2007). cis-Regulatory changes in Kit ligand expression and parallel evolution of pigmentation in sticklebacks and humans. Cell 131: 1179-1189.

Muyle, A. and Gaut, B.S. (2019). Loss of Gene Body Methylation in Eutrema salsugineum Is Associated with Reduced Gene Expression. Mol. Biol. Evol. 36: 155-158.

Nakagawa, S. and Schielzeth, H. (2013). A general and simple method for obtaining R2 from generalized linear mixed-effects models. Methods Ecol. Evol. 4: 133-142.

Nilsson-Ehle, H. (1909). Kreuzungsuntersuchungen an Hafer und Weizen (H. Ohlssons buchdruckerei).

Ortiz-Barrientos, D. (2013). The color genes of speciation in plants. Genetics 194: 39-42.

Osakabe, A., Jamge, B., Axelsson, E., Montgomery, S.A., Akimcheva, S., Kuehn, A.L., Pisupati, R., Lorković, Z.J., Yelagandula, R., Kakutani, T., and Berger, F. (2021). The chromatin remodeler DDM1 prevents transposon mobility through deposition of 
histone variant H2A.W. Nat. Cell Biol. 23: 391-400.

Papareddy, R.K., Páldi, K., Smolka, A.D., Hüther, P., Becker, C., and Nodine, M.D. (2021). Repression of CHROMOMETHYLASE 3 prevents epigenetic collateral damage in Arabidopsis. Elife 10.

Pikaard, C.S. and Mittelsten Scheid, O. (2014). Epigenetic regulation in plants. Cold Spring Harb. Perspect. Biol. 6: a019315.

Qian, S., Wang, Y., Ma, H., and Zhang, L. (2015). Expansion and Functional Divergence of Jumonji C-Containing Histone Demethylases: Significance of Duplications in Ancestral Angiosperms and Vertebrates. Plant Physiol. 168: 1321-1337.

Rajagopalan, R., Vaucheret, H., Trejo, J., and Bartel, D.P. (2006). A diverse and evolutionarily fluid set of microRNAs in Arabidopsis thaliana. Genes Dev. 20: 34073425.

Reinders, J., Wulff, B.B.H., Mirouze, M., Marí-Ordóñez, A., Dapp, M., Rozhon, W., Bucher, E., Theiler, G., and Paszkowski, J. (2009). Compromised stability of DNA methylation and transposon immobilization in mosaic Arabidopsis epigenomes. Genes Dev. 23: 939-950.

Riddle, N.C. and Richards, E.J. (2002). The control of natural variation in cytosine methylation in Arabidopsis. Genetics 162: 355-363.

Robinson, M.D., McCarthy, D.J., and Smyth, G.K. (2010). edgeR: a Bioconductor package for differential expression analysis of digital gene expression data. Bioinformatics 26: $139-140$.

Sasaki, E., Frommlet, F., and Nordborg, M. (2018). GWAS with Heterogeneous Data: Estimating the Fraction of Phenotypic Variation Mediated by Gene Expression Data. G3 8: 3059-3068.

Sasaki, E., Kawakatsu, T., Ecker, J.R., and Nordborg, M. (2019). Common alleles of CMT2 and NRPE1 are major determinants of $\mathrm{CHH}$ methylation variation in Arabidopsis thaliana. PLoS Genet. 15: e1008492.

Sasaki, E., Köcher, T., Filiault, D.L., and Nordborg, M. (2021). Revisiting a GWAS peak in Arabidopsis thaliana reveals possible confounding by genetic heterogeneity. Heredity 127: 245-252.

Saze, H., Shiraishi, A., Miura, A., and Kakutani, T. (2008). Control of Genic DNA Methylation by a jmjC Domain-Containing Protein in Arabidopsis thaliana. Science 319: 462-465.

Schultz, M.D. et al. (2015). Human body epigenome maps reveal noncanonical DNA methylation variation. Nature 523: 212-216.

Shen, X., De Jonge, J., Forsberg, S.K.G., Pettersson, M.E., Sheng, Z., Hennig, L., and Carlborg, Ö. (2014). Natural CMT2 variation is associated with genome-wide methylation changes and temperature seasonality. PLoS Genet. 10: e1004842.

Song, Y., Wu, K., Dhaubhadel, S., An, L., and Tian, L. (2010). Arabidopsis DNA methyltransferase AtDNMT2 associates with histone deacetylase AtHD2s activity. Biochem. Biophys. Res. Commun. 396: 187-192.

Soppe, W.J.J., Jasencakova, Z., Houben, A., Kakutani, T., Meister, A., Huang, M.S., Jacobsen, S.E., Schubert, I., and Fransz, P.F. (2002). DNA methylation controls 
bioRxiv preprint doi: https://doi.org/10.1101/2022.02.09.479810; this version posted February $10,2022$. The copyright holder for this preprint (which was not certified by peer review) is the author/funder, who has granted bioRxiv a license to display the preprint in perpetuity. It is made available under aCC-BY 4.0 International license.

histone $\mathrm{H} 3$ lysine 9 methylation and heterochromatin assembly in Arabidopsis. EMBO J. 21: 6549-6559.

Stegle, O., Parts, L., Piipari, M., Winn, J., and Durbin, R. (2012). Using probabilistic estimation of expression residuals (PEER) to obtain increased power and interpretability of gene expression analyses. Nature Protocols 7: 500-507.

Stephens, M. (2013). A unified framework for association analysis with multiple related phenotypes. PLoS One 8: e65245.

Stroud, H., Do, T., Du, J., Zhong, X., Feng, S., Johnson, L., Patel, D.J., and Jacobsen, S.E. (2014). Non-CG methylation patterns shape the epigenetic landscape in Arabidopsis. Nat. Struct. Mol. Biol. 21: 64-72.

Stroud, H., Greenberg, M.V.C., Feng, S., Bernatavichute, Y.V., and Jacobsen, S.E. (2013). Comprehensive analysis of silencing mutants reveals complex regulation of the Arabidopsis methylome. Cell 152: 352-364.

Takuno, S. and Gaut, B.S. (2012). Body-methylated genes in Arabidopsis thaliana are functionally important and evolve slowly. Mol. Biol. Evol. 29: 219-227.

Villicaña, S. and Bell, J.T. (2021). Genetic impacts on DNA methylation: research findings and future perspectives. Genome Biol. 22: 127.

Wibowo, A., Becker, C., Marconi, G., Durr, J., Price, J., Hagmann, J., Papareddy, R., Putra, H., Kageyama, J., Becker, J., Weigel, D., and Gutierrez-Marcos, J. (2016). Hyperosmotic stress memory in Arabidopsis is mediated by distinct epigenetically labile sites in the genome and is restricted in the male germline by DNA glycosylase activity. Elife 5.

Xing, H.-L., Dong, L., Wang, Z.-P., Zhang, H.-Y., Han, C.-Y., Liu, B., Wang, X.-C., and Chen, Q.-J. (2014). A CRISPR/Cas9 toolkit for multiplex genome editing in plants. BMC Plant Biol. 14: 327.

Yeaman, S. et al. (2016). Convergent local adaptation to climate in distantly related conifers. Science 353: 1431-1433.

Yu, J., Pressoir, G., Briggs, W.H., Vroh Bi, I., Yamasaki, M., Doebley, J.F., McMullen, M.D., Gaut, B.S., Nielsen, D.M., Holland, J.B., Kresovich, S., and Buckler, E.S. (2006). A unified mixed-model method for association mapping that accounts for multiple levels of relatedness. Nat. Genet. 38: 203-208.

Zhang, X., Henriques, R., Lin, S.-S., Niu, Q.-W., and Chua, N.-H. (2006). Agrobacteriummediated transformation of Arabidopsis thaliana using the floral dip method. Nat. Protoc. 1: 641-646.

Zhang, Y., Jang, H., Xiao, R., Kakoulidou, I., Piecyk, R.S., Johannes, F., and Schmitz, R.J. (2021). Heterochromatin is a quantitative trait associated with spontaneous epiallele formation. Nat. Commun. 12: 6958.

Zheng, X., Pontes, O., Zhu, J., Miki, D., Zhang, F., Li, W.-X., lida, K., Kapoor, A., Pikaard, C.S., and Zhu, J.-K. (2008). ROS3 is an RNA-binding protein required for DNA demethylation in Arabidopsis. Nature 455: 1259-1262.

Zhong, X., Hale, C.J., Law, J.A., Johnson, L.M., Feng, S., Tu, A., and Jacobsen, S.E. (2012). DDR complex facilitates global association of RNA polymerase $\mathrm{V}$ to promoters and evolutionarily young transposons. Nat. Struct. Mol. Biol. 19: 870-875. 


\section{Supplemental figures}

A
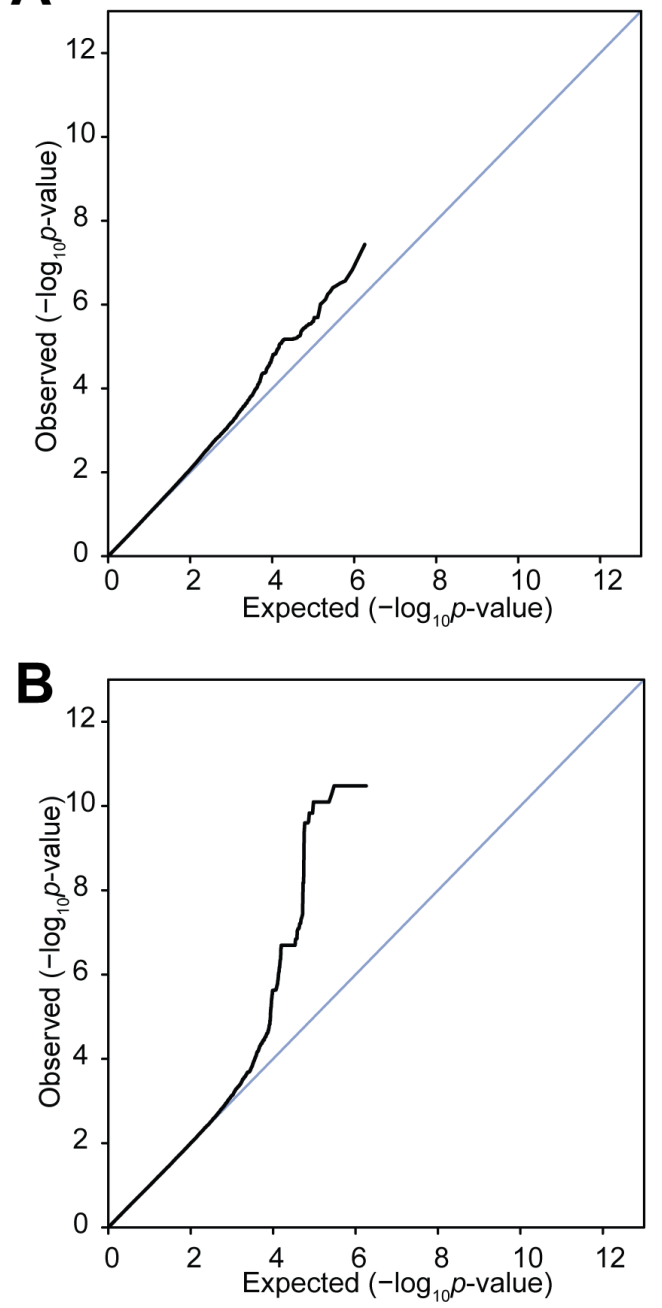
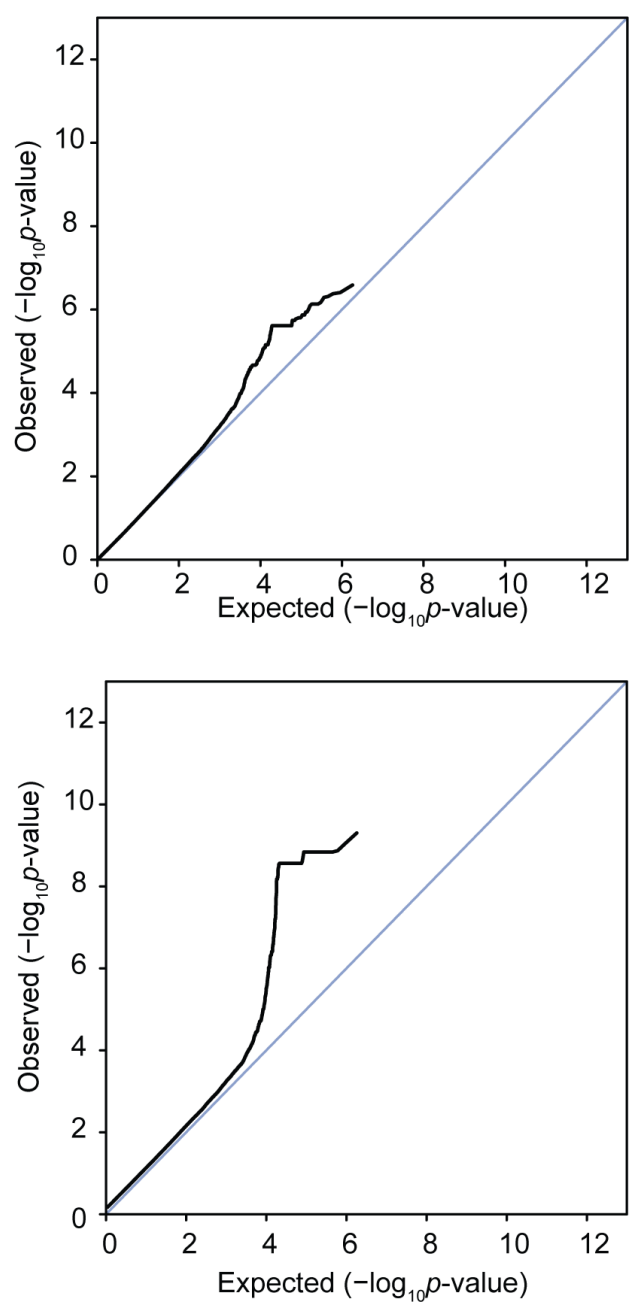

Fig S1. Distribution of $p$-values for two GWAS models. $Q Q$ plots for univariate models for $\mathrm{mCHG}$ levels (A) and conditional models for $\mathrm{mCHG}_{\mid \mathrm{mCHH}}$ (B) in RdDM-targeted transposons (left) and CMT2targeted transposons (right). 
A
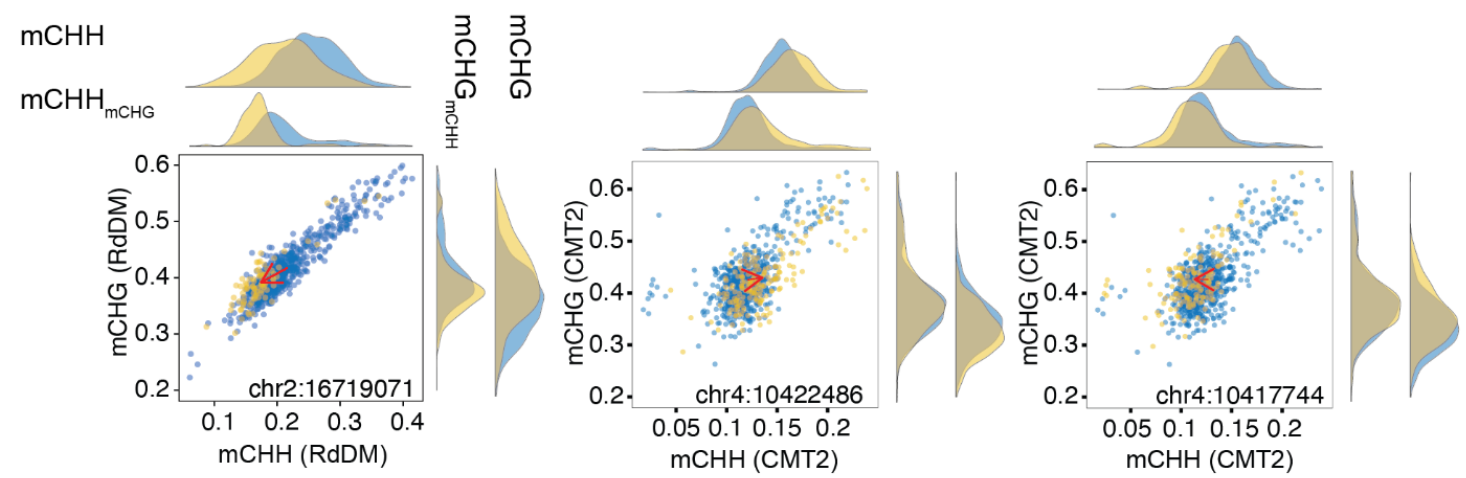

B
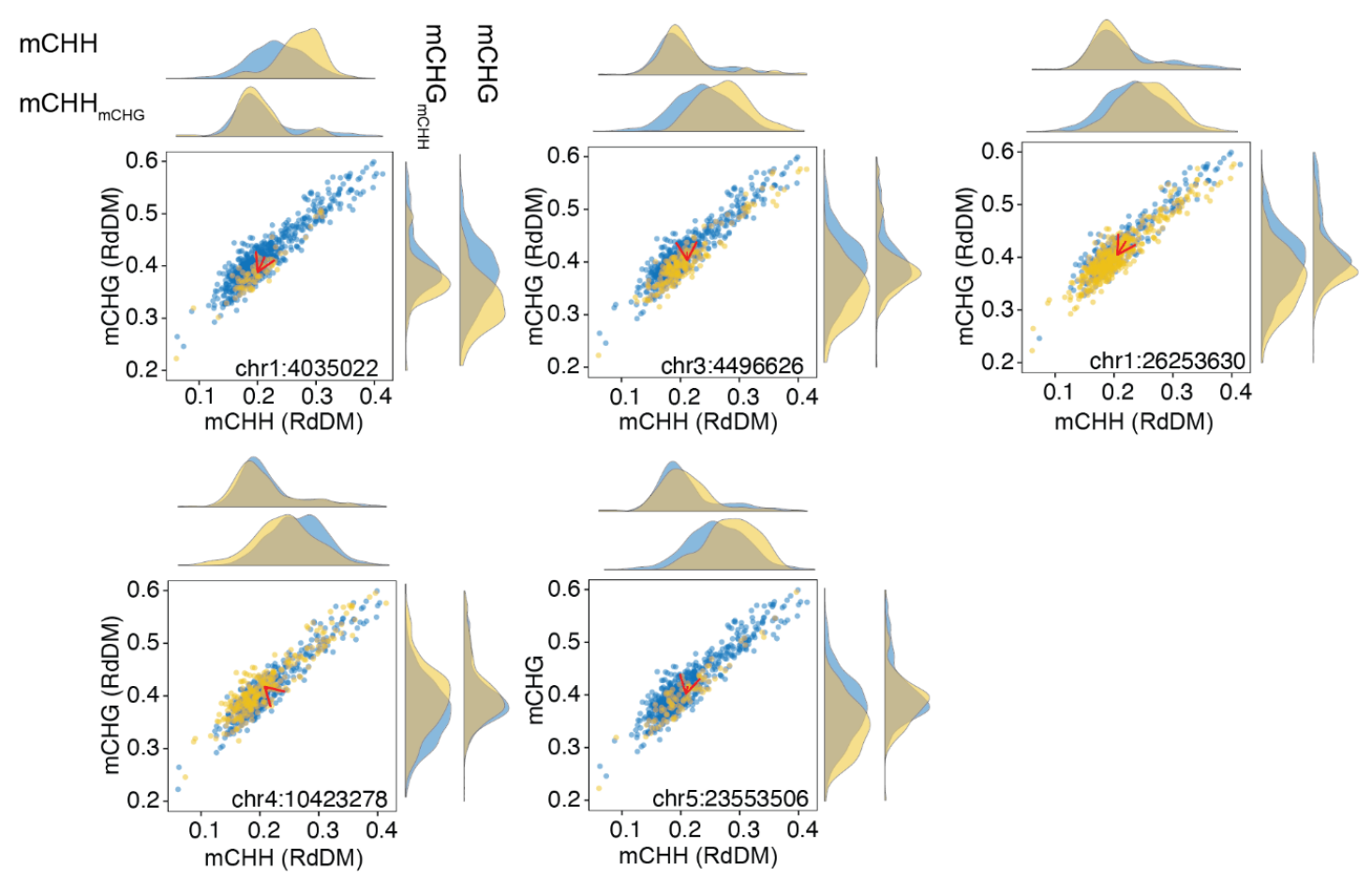

C
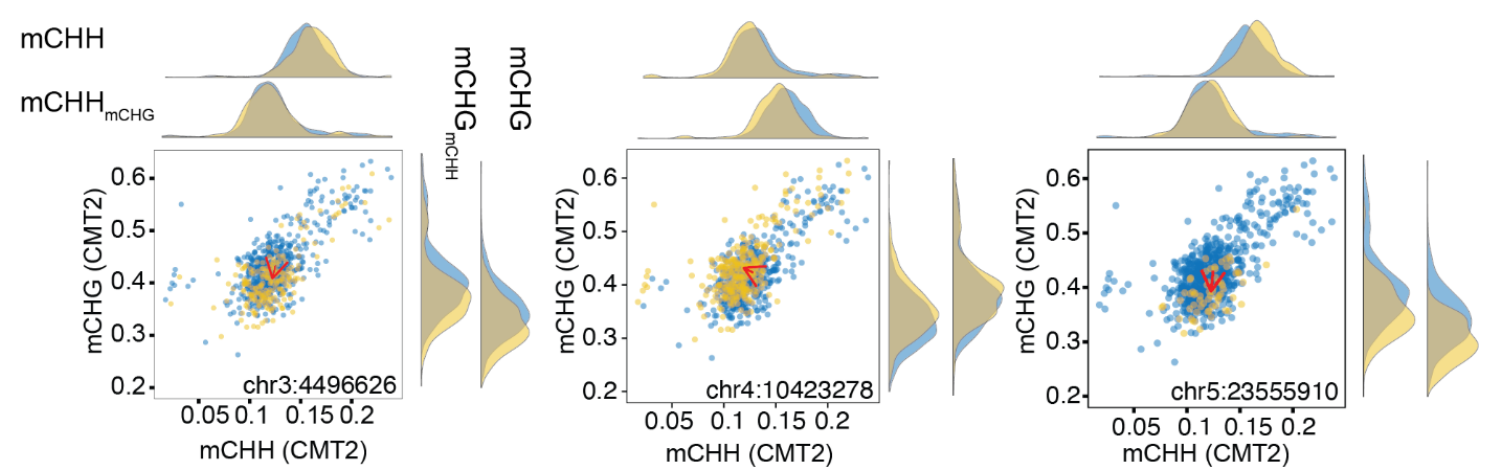

Fig S2. Distribution of phenotypes and the allelic effects. The scatter plots illustrate the allelic effects on $\mathrm{mCHH}$ (A from Sasaki et al., 2019) and $\mathrm{mCHG}_{\mid \mathrm{mCHH}}$ in RdDM- (B) and CMT2-targeted transposons (C). Marginal phenotypic distributions for the reference and the alternative allele are plotted in blue and yellow, for univariate and conditional distributions. Red vectors show shifts of the mean value from the reference to the alternative allele in 2-dimensional phenotype space. 

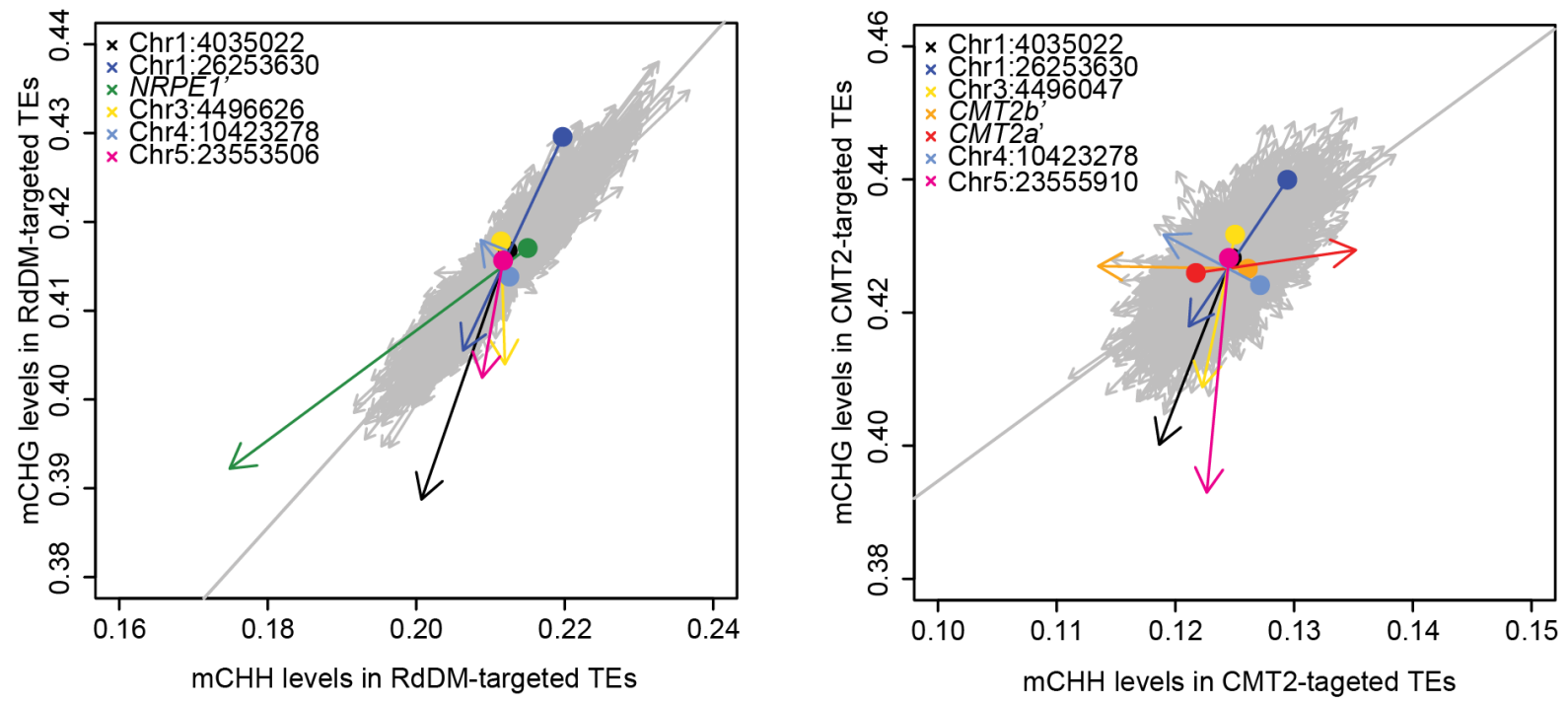

Fig S3. Comparison of the allelic effects on $\mathrm{mCHH}$ and $\mathbf{m C H G}$. Each arrow shows the mean value shift of $\mathrm{mCHH}$ and $\mathrm{mCHG}$ levels from reference to the alternative allele. Left and right plot correspond to RdDM- and CMT2-targeted transposons, respectively. Gray arrows correspond to the genetic effects of randomly sampled SNPs, keeping the allele frequency of each tested SNP (x 3000 for each allele). Diagonal lines correspond to linear regression lines of $\mathrm{mCHH}$ and $\mathrm{mCHG}$. 
bioRxiv preprint doi: https://doi.org/10.1101/2022.02.09.479810; this version posted February 10, 2022. The copyright holder for this

preprint (which was not certified by peer review) is the author/funder, who has granted bioRxiv a license to display the preprint in perpetuity. It is made available under aCC-BY 4.0 International license.

A

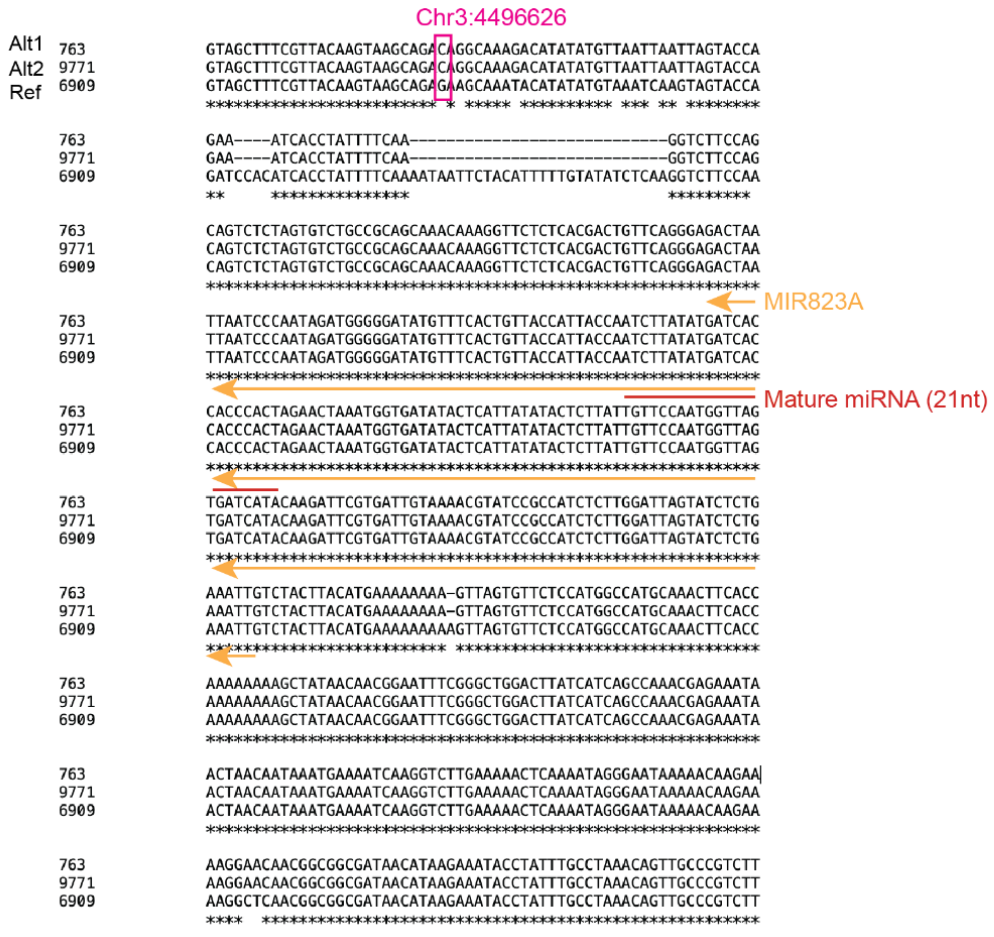

B

C
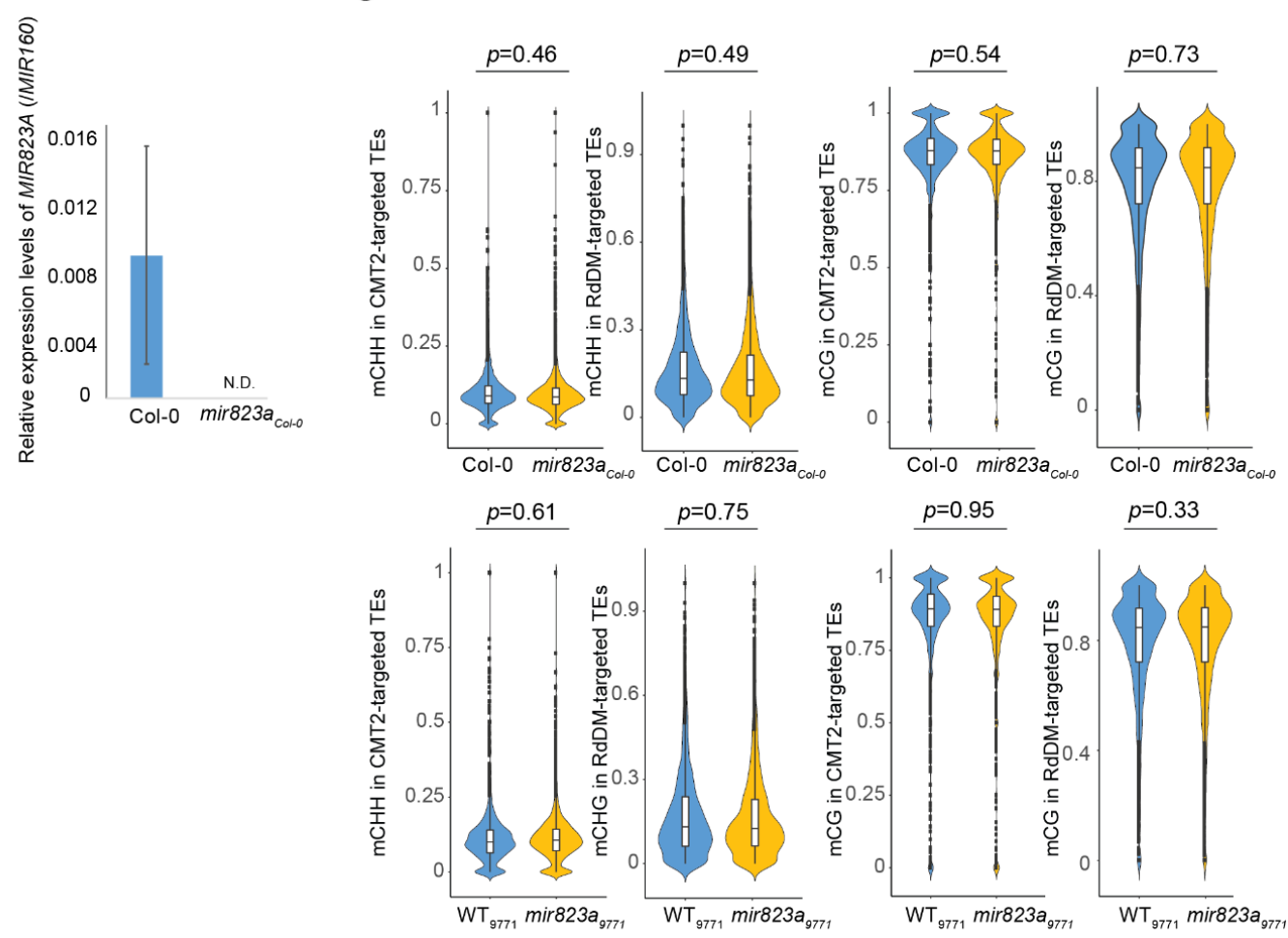

Fig S4. Characterization of MIR823A. (A) Sanger sequences around MIR823A region for lines carrying reference allele (Col-0) and the alternative allele (763, 9771). (B) MIR823A expression in a CRISPR/CAS9 mir823a mutant (Col-0) by qRT-PCR. Error bar shows standard deviation ( $n=3)$. (C) Effects of MIR823A on mCHH and mCG levels in RdDM and CMT2-targeted transposons in mir823a Col-0 and 9771 background. 
A

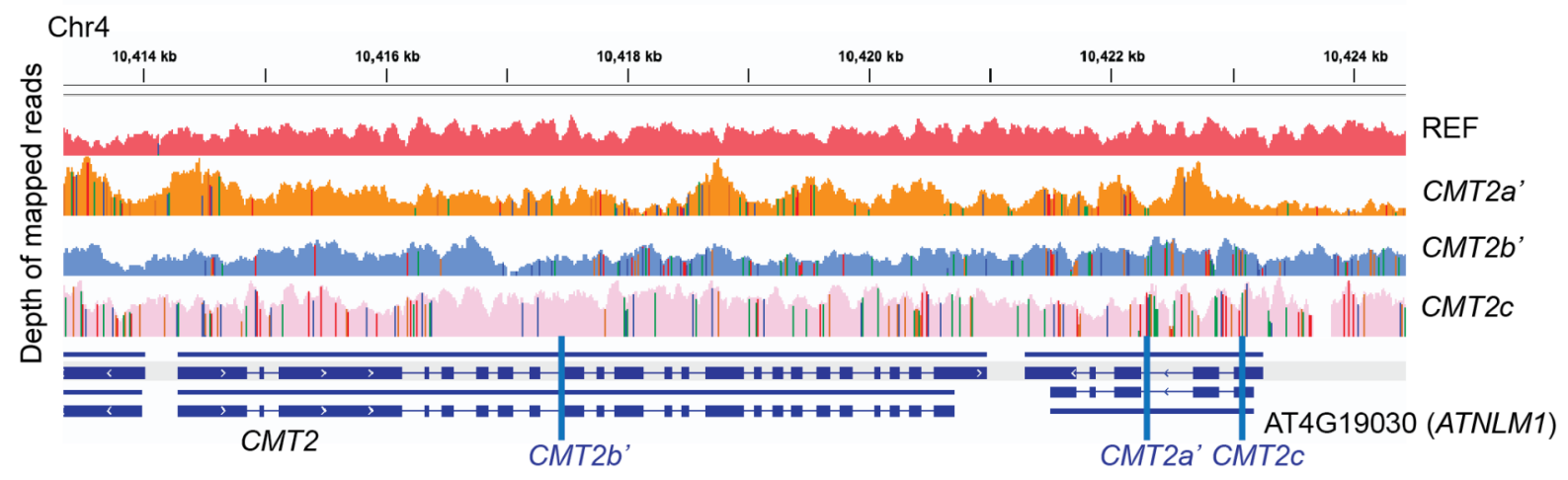

B
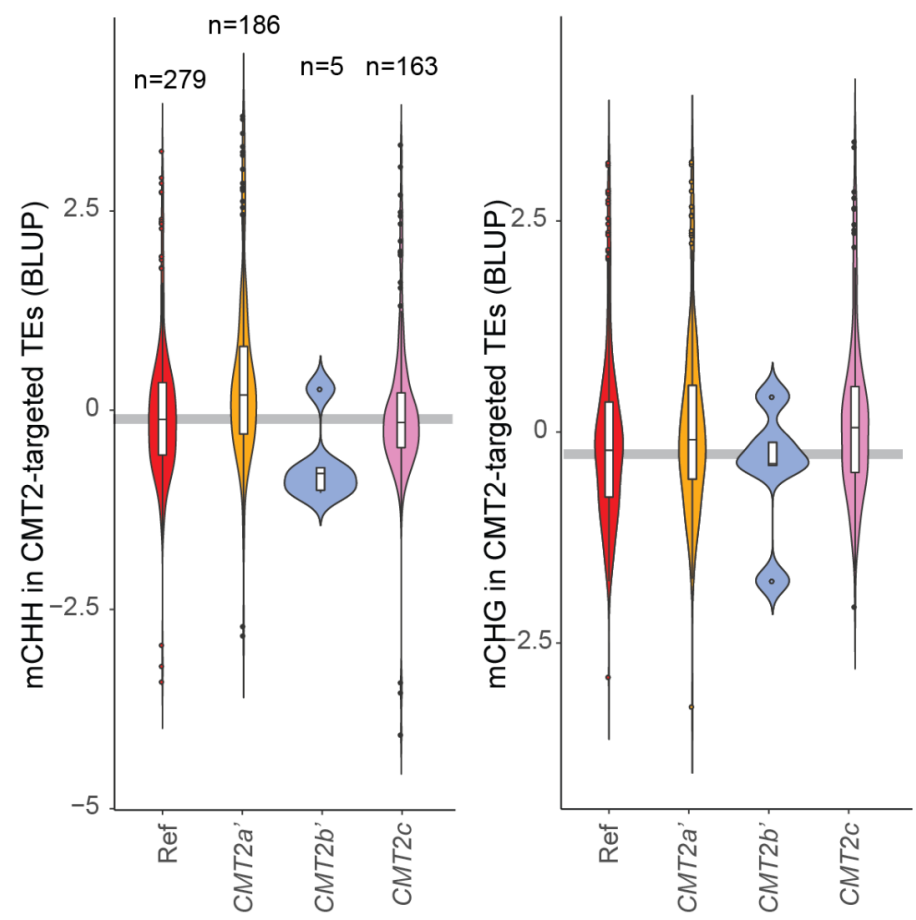

Fig S5. The effects of CMT2 alleles on non-CG methylation. (A) Genome structure of three CMT2 alleles associated with non-CG methylation variation. The CMT2 region was illustrated by mapped short-read DNA-seq data (IGV browser) for reference line (Col-0), CMT2a' (10018), CMT2b' (6969), and CMT2C (10023). Vertical colored lines in the IGV plots indicate SNPs. (B) The allelic effects on genome-wide average $\mathrm{mCHH}$ and $\mathrm{mCHG}$ levels in $\mathrm{CMT} 2$-targeted transposons. Only lines carrying one allele were compared with the reference line. Horizontal gray lines show median values of the reference lines. 
A

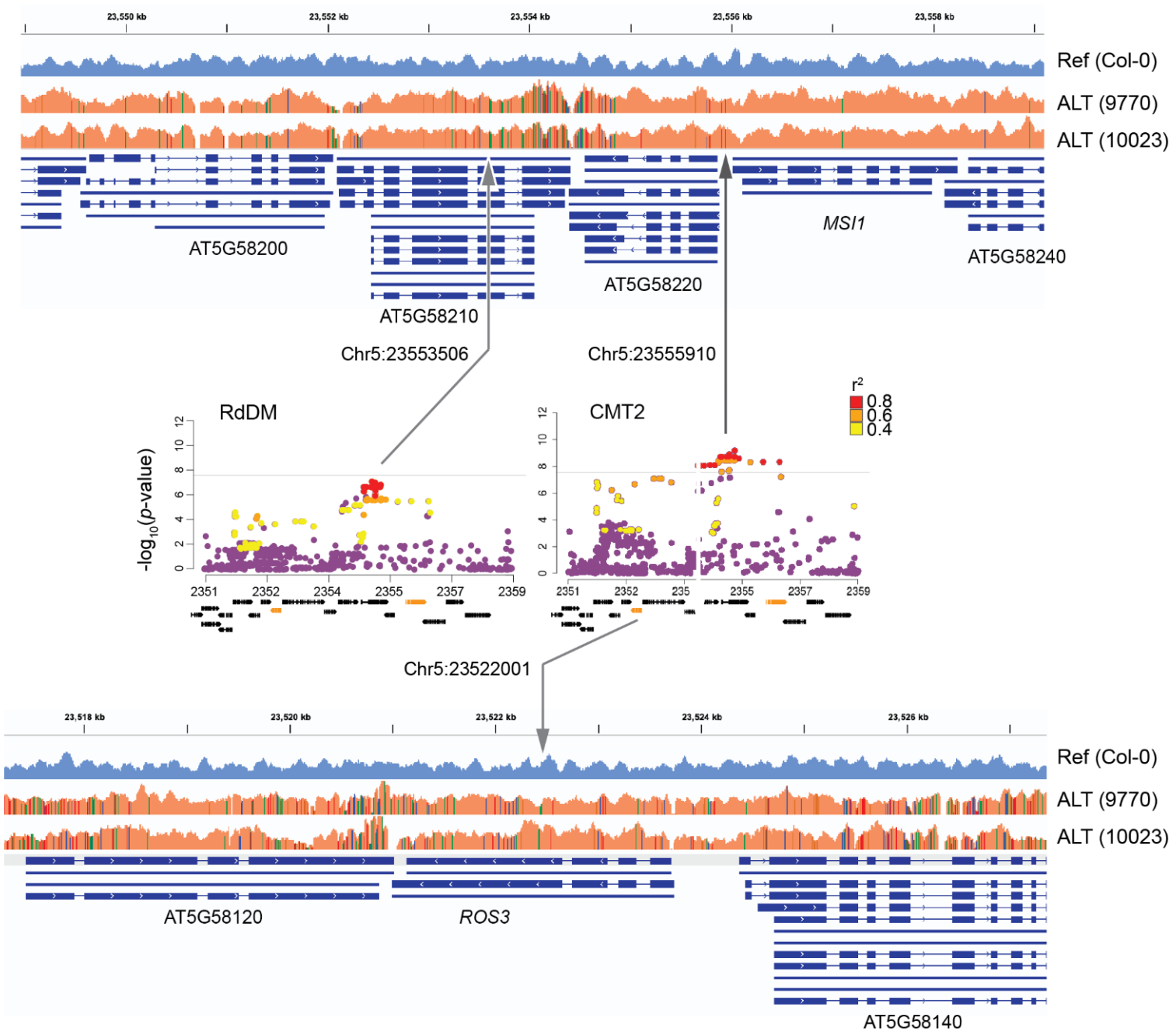

B
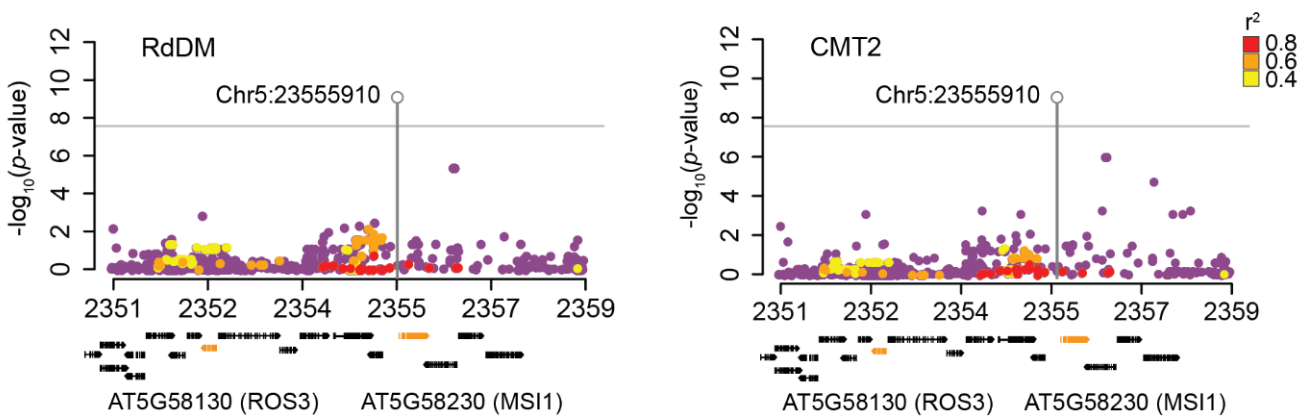

Fig S6. Genetic variation around MSI1 and ROS3. (A) Zoom-in Manhattan plots (Fig 3) and the genome structure around Chr5:23553506, 23555910 (top), and 23522001 (bottom) illustrated by mapped short-read DNA-seq data (IGV browser). Vertical colored lines in the IGV plots show SNPs. (B) Conditional GWAS for $\mathrm{mCHG}$ in RdDM- and CMT2-targeted transposons. $\mathrm{mCHH}$ and Chr5:23555910 were both used as co-factors. Gray vertical lines indicate the Chr5:2355910 position, and horizontal lines show the genome-wide significance ( $p=0.05$ by Bonferroni correction). $r^{2}$ was calculated from chr5:23553506 and chr5:23555910 for mCHGRdDM and $\mathrm{mCHG}_{\mathrm{CMT}}$, respectively. 
bioRxiv preprint doi: https://doi.org/10.1101/2022.02.09.479810; this version posted February 10, 2022. The copyright holder for this preprint (which was not certified by peer review) is the author/funder, who has granted bioRxiv a license to display the preprint in perpetuity. It is made available under aCC-BY 4.0 International license.

A

ALT (5984) EFISALPFQEYSDPRSGILNIATKLPEGLLKPDLGPKTYIAYGTSDELGRGDSVTKLHCD REF (6909) EFISALPFOEYSDPRSGILNIATKLPEGLLKPDLGPKTYVAYGTSDELGRGDSVTKLHCD $* * * * * * * * * * * * * * * * * * * * * * * * * * * * * * * * * * * * * * *: * * * * * * * * * * * * * * * * * * * *$

MSDAVNILMHTAEVTVSVEQRSAIADLKQKHKQQNEKELQEQNGLEEEEVVSDEFVVYDE MSDAVNILMHTAEVTLSEEQRSAIADLKQKHKQQNEKELQEQNGLEEEEVVSDEIVVYDE

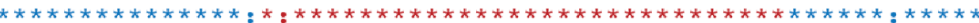
Chr1:4035683, 4035690

TGGALWDIFRREDVPKLEEYLRKHCIEFRHTYCSRVTKVYHPIHDOSYFLTVEHKRKLKA TSGALWDIFKREDVPKLEEYLRKHCIEFRHTYCSRVTKVYHPIHDQSYFLTVEHKRKLKA ****t***t*

EFGIEPWTFVQKLGEAVFIPAGCPHQVRNLKSCTKVAVDFVSPENIDECLRLTDEFROLP EFGIEPWTFVQKLGEAVFIPAGCPHQVRNLKSCTKVAVDFVSPENIDECLRLTDEFRQLP *****************************************************************

KNHKAREDKLEIKKMVIYAVEQALKEVETLLLDRS

JMJ domain 614-837aa KNHKAREDKLEIKKMVIYAVEQALKEVETLLLDRS Coiled coil 677-708aa $\star * * * * * * * * * * * * * * * * * * * * * * * * * * * * * * * * * * * * * * *$

B

O.sativa
C.quinoa
B.stricta
A.lyrata
AT1G11950_CDS_6909
AT1G11950_CDS_5984
S.lycopersicum
G.max
T.cacao
V.vinifera

O.sativa
C.quinoa
B.stricta
A.lyrata
AT1G11950_CDS_6909
AT1G11950_CDS_5984
S.lycopersicum
G.max
T.cacao
V.vinifera

YMRGRRHPMTFWPEMLKLKDWPPSSMFDORLPRHGAEFITALPFPEYTDPRYGPLNLAVR 667 YTKCQFDP-YYWPLLLKLKDWPPSTEFDONLPRHGAEFVQALPFKEYTHHLSGILNLASK 734 YSKGRRYA-NFWPEMLKLKDWPPSDKFENLLPRHCDEFISALPFOEYSNPRSGILNIAAK 634 YSKGRRYD-NFWPEMLKLKDWPPSDKFENLLPRHCDEFISALPFQEYSDPRSGILNIATK 625 YSKGRTYE-NFWPEMLKLKDWPPSDKFENLLPRHCDEFISALPFOEYSDPRSGILNIATK 624 YSKGRTYE-NFWPEMLKLKDWPPSDKFENLLPRHCDEFISALPFQEYSDPRSGILNIATK 624 YTEGRRYE-NLWPEMLKLKDWPPSDKFEKVLPRHCDEFISALPFOEYTDPRIGILNLAVK 570 YTQGRTYR-NLWPEMLKLKDWPPSHKFEDLLPRHYDEFIRCLPFQEYSDPRAGILNLAVK 585 YMEGRRYD-NFWPEMLKLIKDWPPSNEFEDLLPRHCDEFISALPFOEYSDPRSGILNLAVK 621 YTEGRSYD-NLWPEMLKLKDWPPSDKFENLLPRHCDEFISALPFOEYTDPRAGFLNLAVK 426 *. : $\quad * *: * * * * * * * *: . * * * * * *: . * * * *: . \quad * * *: *:$

LPAGVLKPDLGPKTYIAYGCYEELGRGDSVTKLHCDMSDAVNILMHTAEVSYDTEOLDKI 727 LPTDYLKPDLGPKTYIAYGVAOELGRGDSVTKLHCDMSDAVNILTHTAETKLNASOLQRI 794 LPEGLLKPDLGPKTYIAYGTSDELGRGDSVAKLHCDMSDAVNILMHTTEVTITEEORTAI 694 LPEGLLKPDLGPKTYIAYGTSDELGRGDSVTKLHCDMSDAVNILMHTAEVTLSEEOMSAI 685 LPEGLLKPDLGPKTYVAYGTSDELGRGDSVTKLHCDMSDAVNILMHTAEVTLSEEORSAI 684 LPEGLLKPDLGPKTYIAYGTSDELGRGDSVTKLHCDMSDAVNILMHTAEVTVSVEOKSAI 684 LPAGVIKPDLGPKTYIAYGLSEELGRGDSVTKLHCDMSDAINILTHTAEMAITDEORSAI 630 LPPHVLKPDLGPKTYIAYGIKEELGRGDSVTKLHCDMSDAVNILTHTAEVTLTDEONCVI 645 LPPGVLKPDLGPKTYIAYGIAEELGRGDSVTKLHCDMSDAVNILTHIADVALSKEOLAAI 681 LPNTILKPDLGPKTYIAYGIAEELGRGDSVTKLHCDMSDAVNILTHTAEVVLDDNORLAV 486 $* * \quad: * * * * * * * *: * * * \quad: * * * * * * * *: * * * * * * * *: * * * *:: \quad$ : $\quad:$

Chr1:4035683, 4035690

Fig S7. Characterization of JMJ26 allele. Predicted amino acid sequences around jmjC domain of reference (6909) and the alternative allele (DralV 6-22)(A) and the conserved region (B). Orange bars indicate nonsynonymous mutations associated with $\mathrm{mCHG}_{\mid \mathrm{mCHH}}$ in RdDM-targeted TEs. The sequences were predicted based on polymorphism data provided by the 1001 genome project. The domain information follows the TAIR10 annotation. 
bioRxiv preprint doi: https://doi.org/10.1101/2022.02.09.479810; this version posted February 10, 2022. The copyright holder for this preprint (which was not certified by peer review) is the author/funder, who has granted bioRxiv a license to display the preprint in perpetuity. It is made available under aCC-BY 4.0 International license.

A
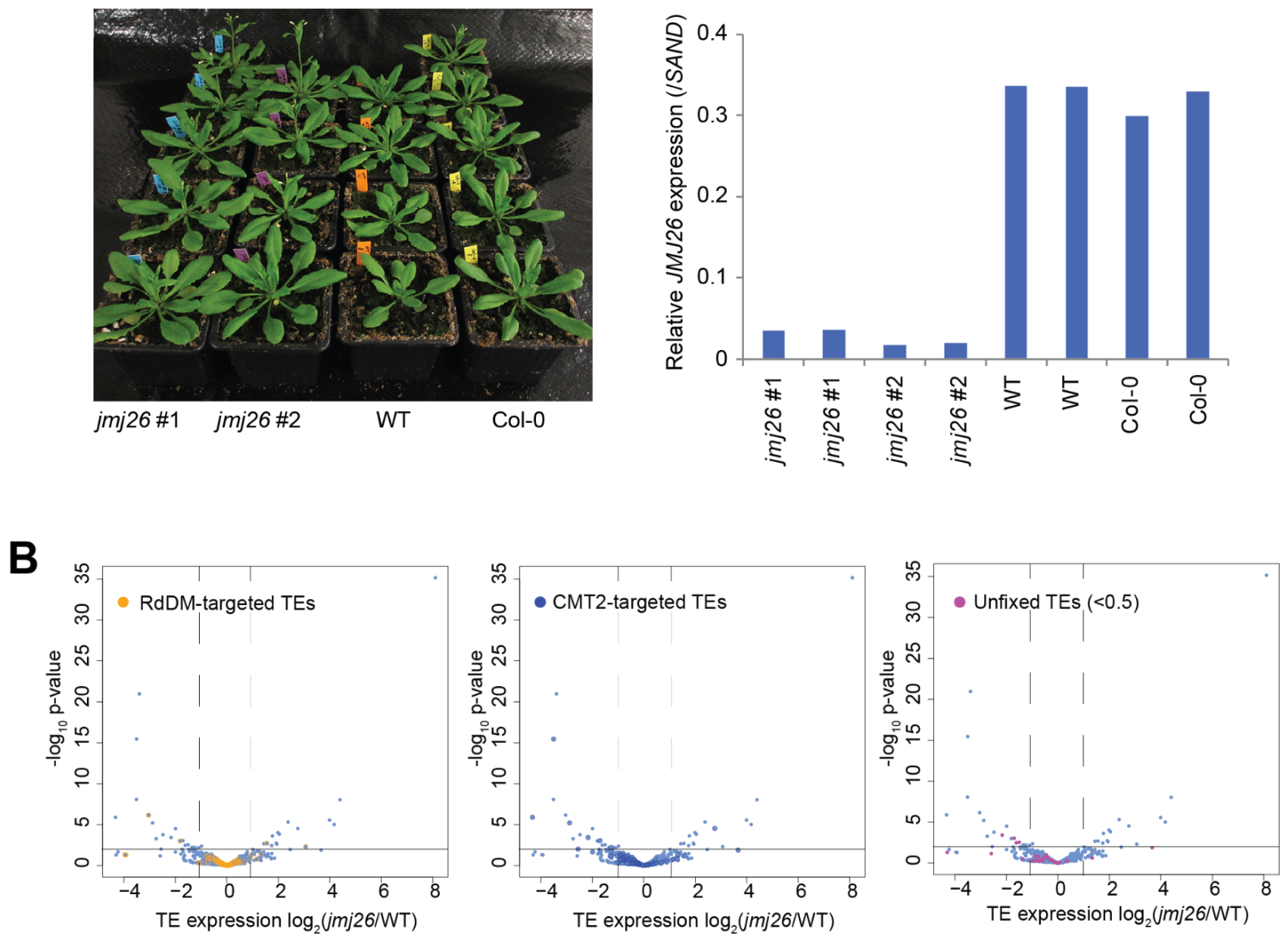

C

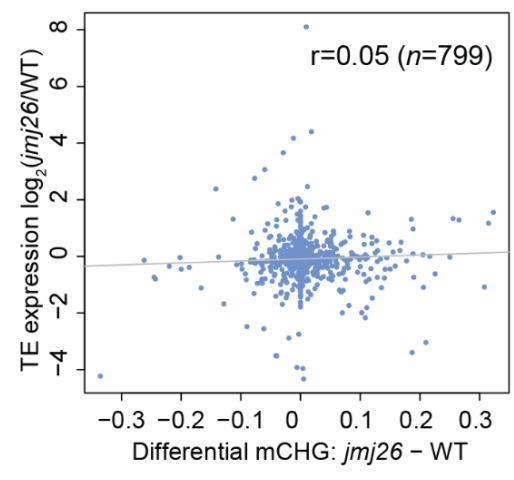

Fig S8. Molecular phenotypes of $j \mathbf{j m j 2 6}$. (A) Characterization of loss-of-function mutants, jmj26. Both \#1 and \#2 were jmj26 homozygous lines isolated from SALKseq_069498.1 and propagated separately. WT is a segregated line carrying active JMJ26 in the same stock. Morphology of jmj26 and Col-0 (left) and JMJ26 expression in leaves of jmj26 and the wild type (right). Expression was measured by qRTPCR. (B) Volcano plots show the effects on transposon transcripts highlighted RdDM- and CMT2targeted transposons. (C) The scatter plot shows the effect of $\mathrm{mCHG}$ methylation on transposon transcripts. The gray line shows the linear regression line. 
bioRxiv preprint doi: https://doi.org/10.1101/2022.02.09.479810; this version posted February 10, 2022. The copyright holder for this preprint (which was not certified by peer review) is the author/funder, who has granted bioRxiv a license to display the preprint in perpetuity. It is made available under aCC-BY 4.0 International license.

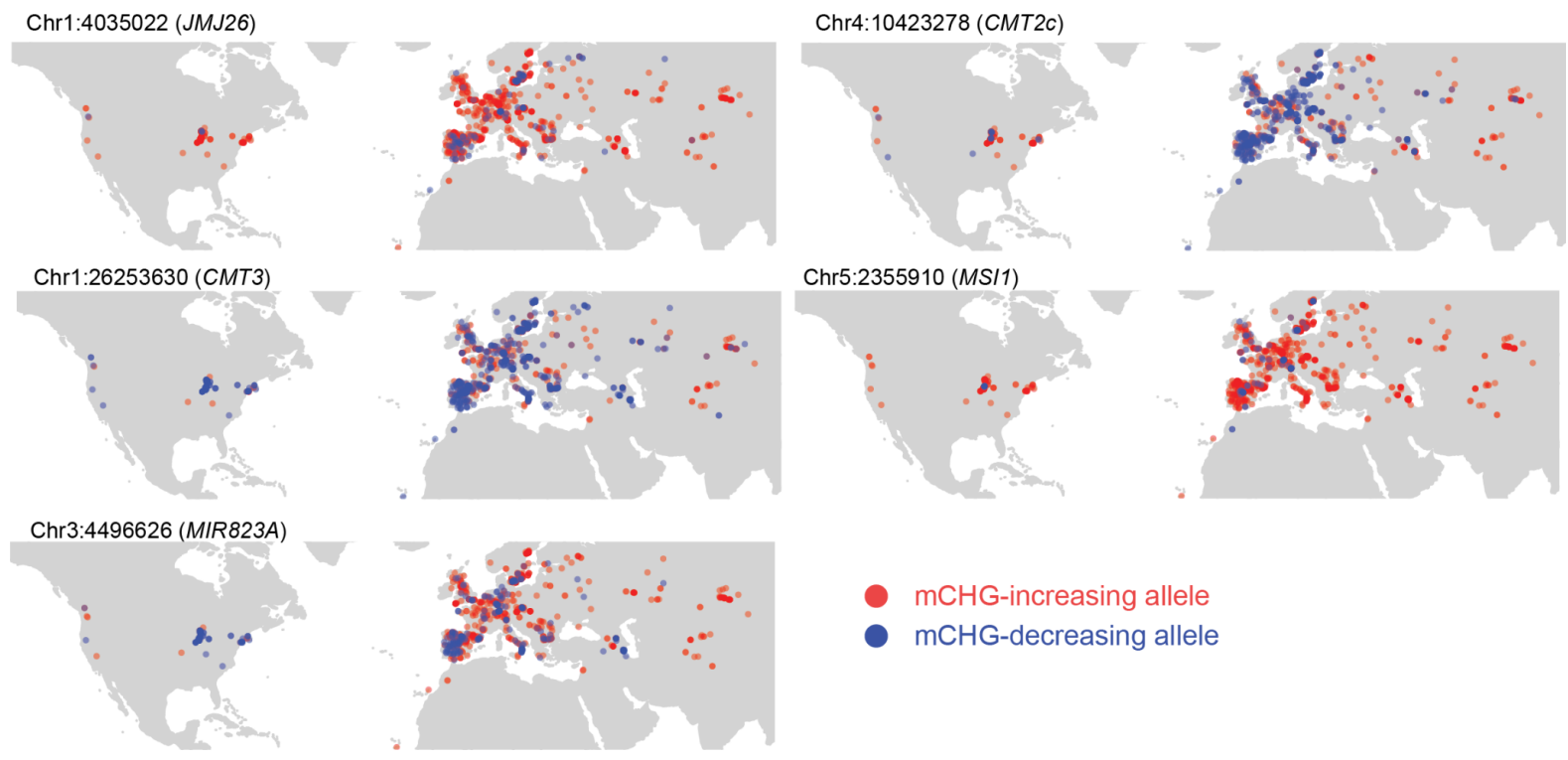

Fig S9. Geographical distribution of mCHG-decreasing alleles. The plots show the origin of lines carrying $\mathrm{mCHG}$-increasing or decreasing alleles. 
A

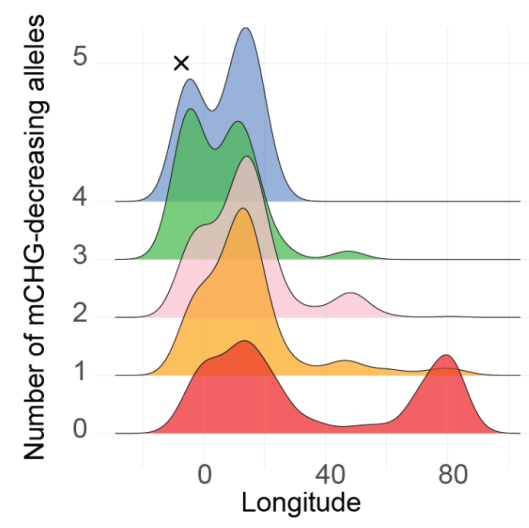

C

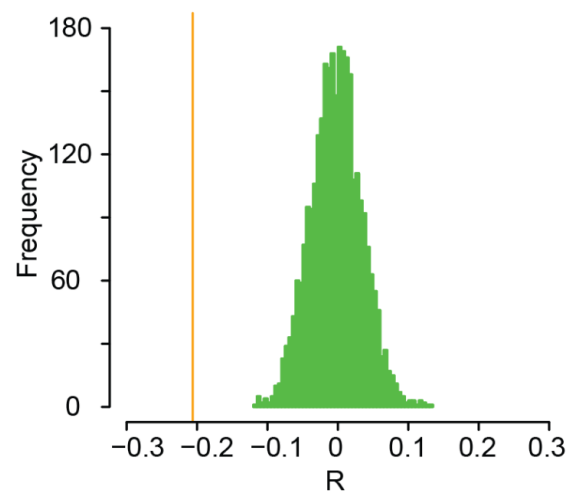

B

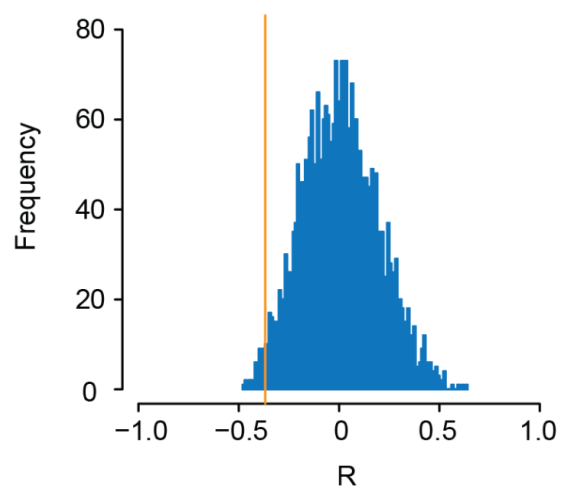

D

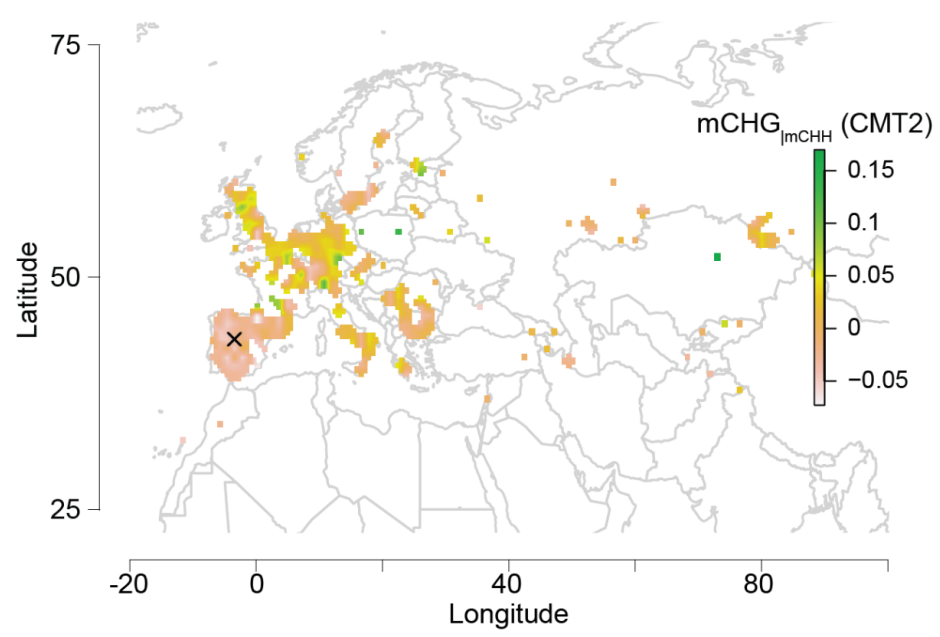

Fig. S10. Geographical distribution of cumulative mCHG-decreasing alleles. (A) Longitudinal frequencies of cumulative mCHG-decreasing alleles (corresponding to five alleles in Fig. 6A). (B) The histogram shows how the cumulative mCHG-decreasing allele number is correlated with longitude of the origins. The blue histogram shows permuted Pearson's correlation coefficients (R) between numbers of $\mathrm{mCHG}$-decreasing alleles and longitude of the origin, maintaining the allele frequencies. The permutation tests were repeated 3000 times for 971 lines ranging from longitude $-20^{\circ}$ to $100^{\circ}$. The orange vertical line indicates the observed value. (C) Histogram similarly shows permuted Pearson's correlation coefficients between number of $\mathrm{mCHG}$-decreasing alleles and NRPE1' genotype. The orange line is the observation. $\mathrm{mCHH}$-increasing and decreasing NRPE1' alleles are 0 and 1, respectively. (D) The geographic distribution of $\mathrm{mCHG}_{\mid \mathrm{mCHH}}$ levels in $\mathrm{CMT2}$-targeted transposons. 
A

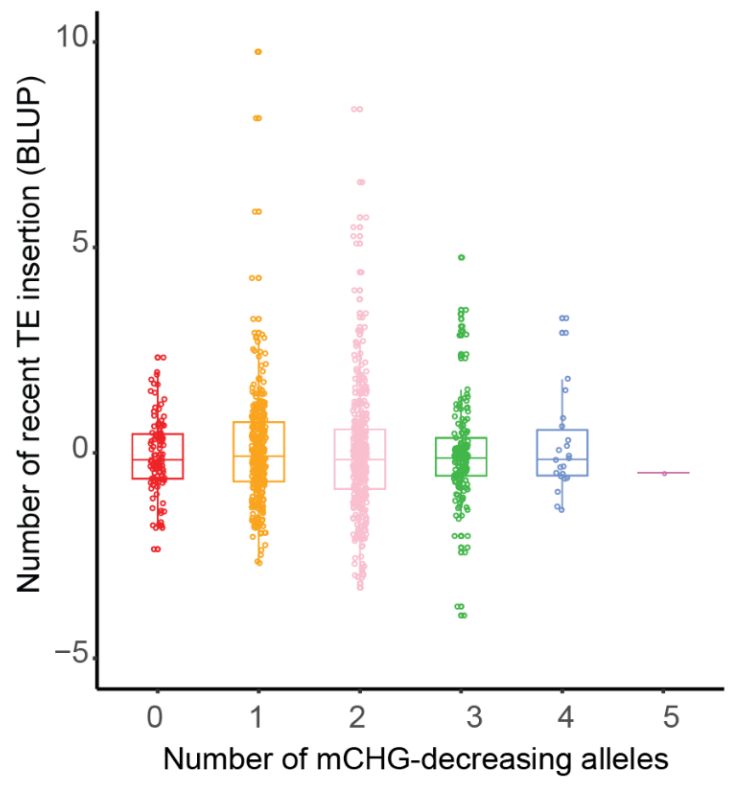

B

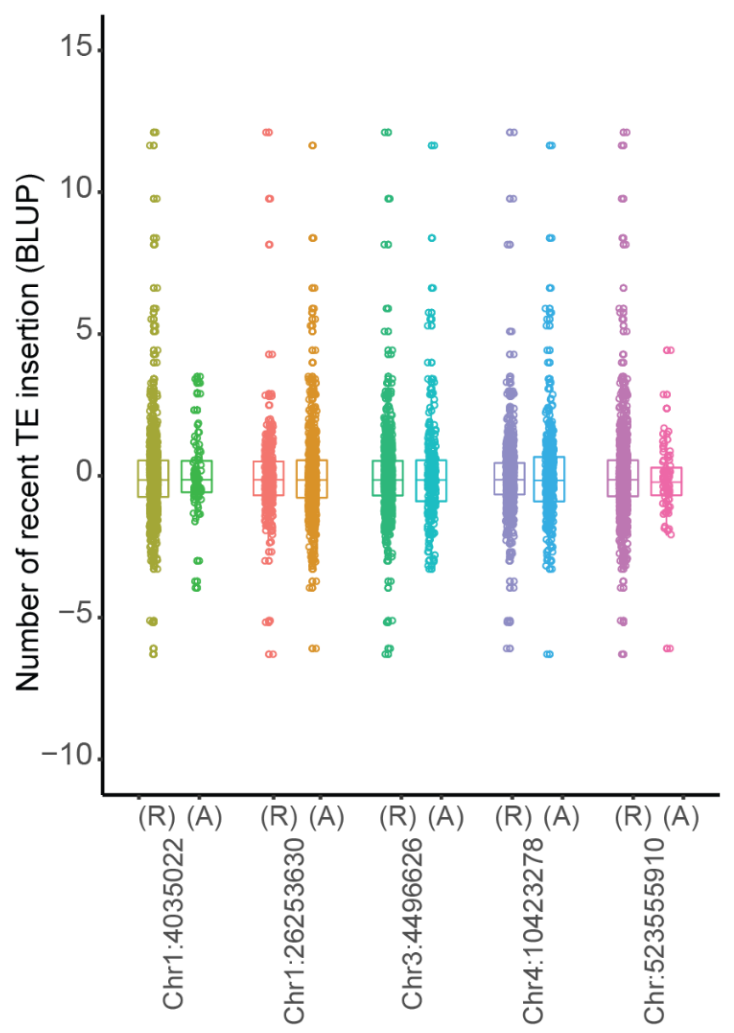

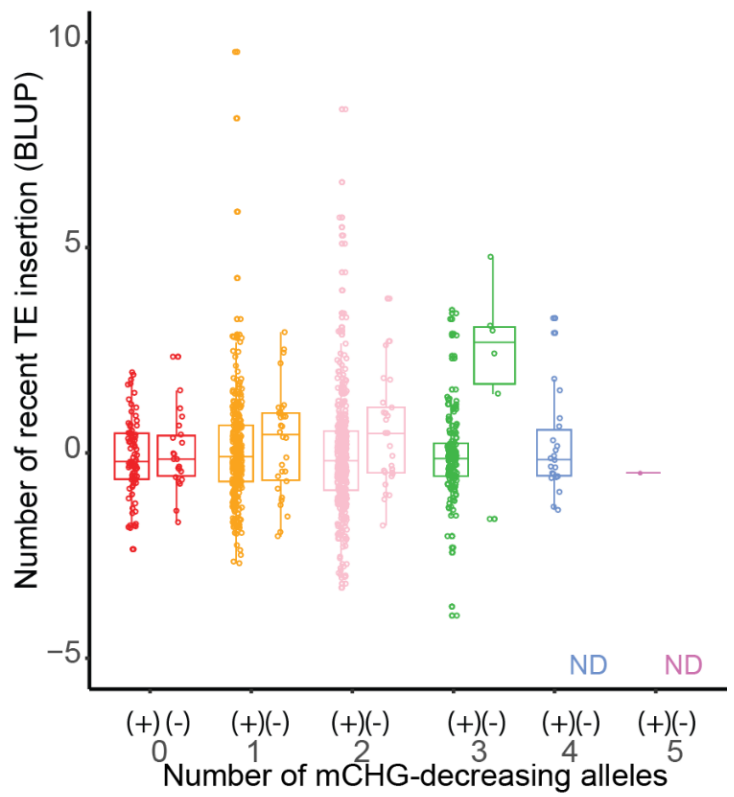

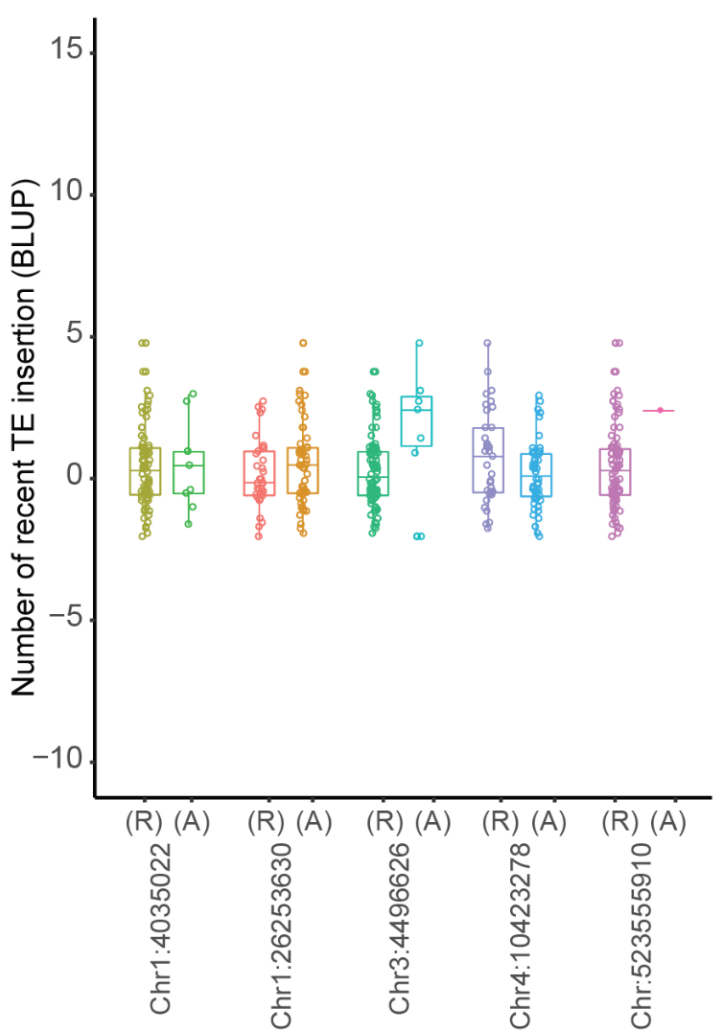

Fig 11. Function of $\mathrm{mCHG}$-decreasing alleles on transposon activities. (A) Effects of the cumulative $\mathrm{mCHG}$-decreasing alleles in whole populations (left) and combination with NRPE1' allele (right). $(+)$ and $(-)$ are lines carrying NRPE1' reference $(\mathrm{mCHH}$-increasing) and the alternative ( $\mathrm{mCHH}$-decreasing) alleles, respectively. (B) Effects of the five major mCHGdecreasing alleles with NRPE1' reference (left) and the alternative (right) populations. $(R)$ and (A) are reference and the alternative alleles, respectively. 
bioRxiv preprint doi: https://doi.org/10.1101/2022.02.09.479810; this version posted February 10,2022 . The copyright holder for this

preprint (which was not certified by peer review) is the author/funder, who has granted bioRxiv a license to display the preprint in perpetuity. It is made available under aCC-BY 4.0 International license.

A

RdDM-targeted TEs
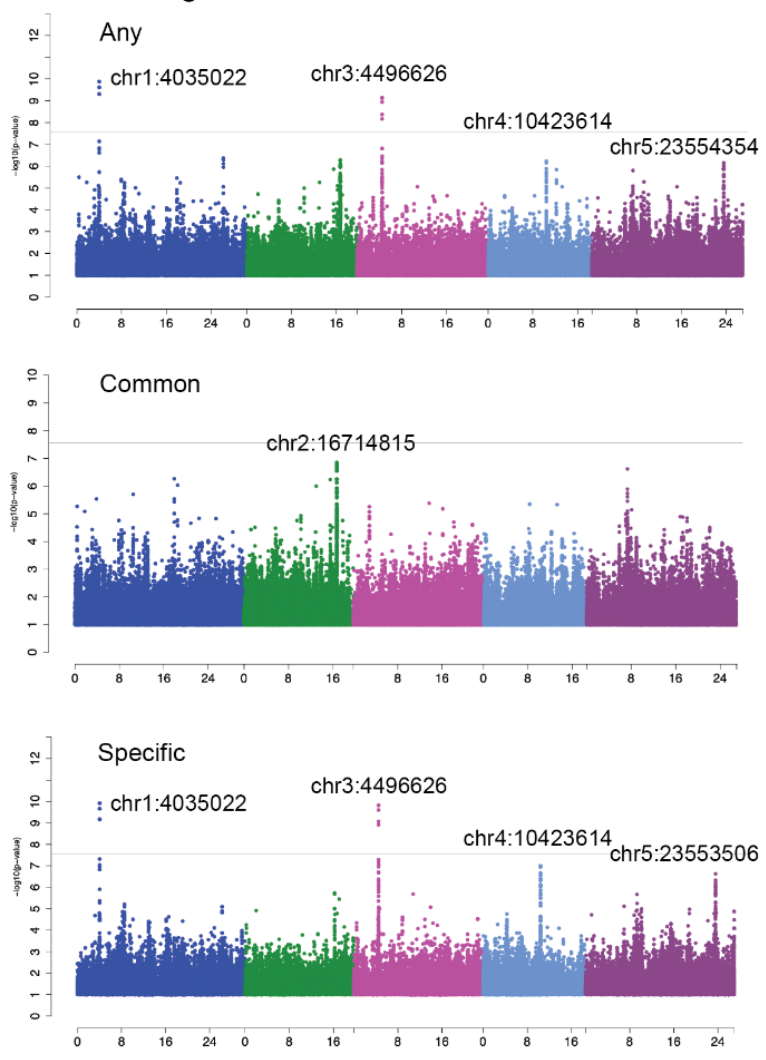

CMT2-targeted TEs

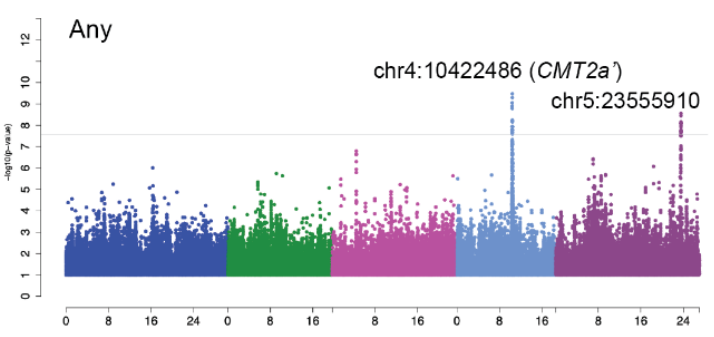

Common

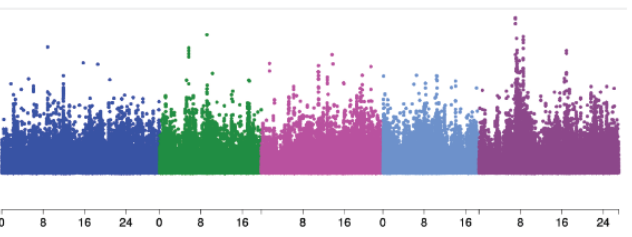

Specific

chr4:10423278

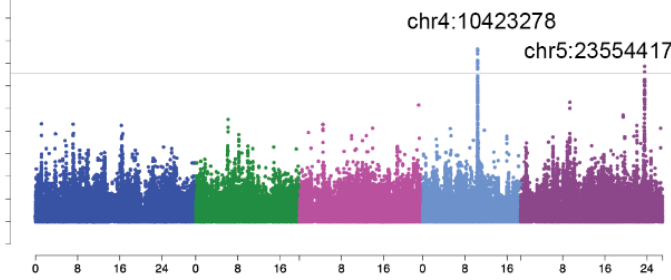

B

RdDM-targeted TEs
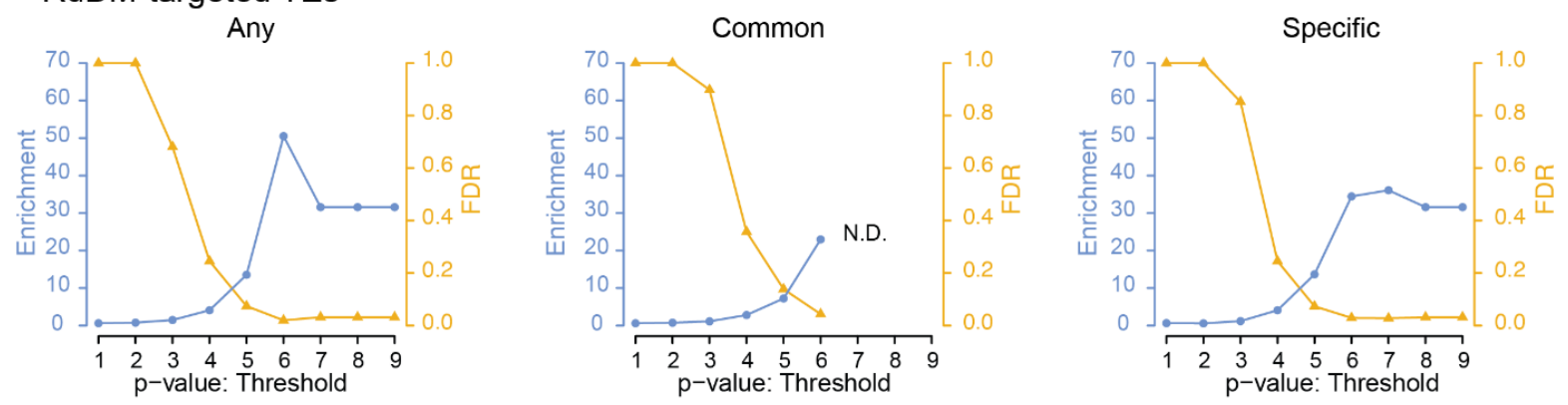

CMT2-targeted TEs
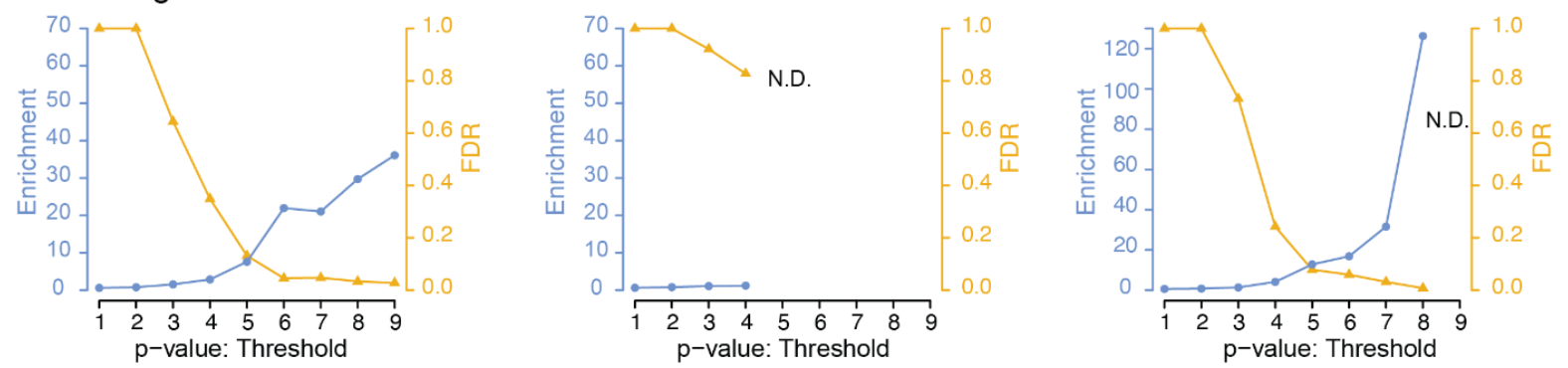

Fig S12. The genetic basis of $\mathrm{mCHG}$ and $\mathrm{mCHH}$ analyzed by MTMM. (A) Manhattan plots for any, common, specific SNP effects on $\mathrm{mCHG}$ and $\mathrm{mCHH}$ in RdDM and CMT2-targeted transposons (see Methods). Vertical lines correspond to genome-wide significance ( $p=0.05$ by Bonferroni correction). (B) Enrichment of a priori genes and FDR for each GWAS result. 

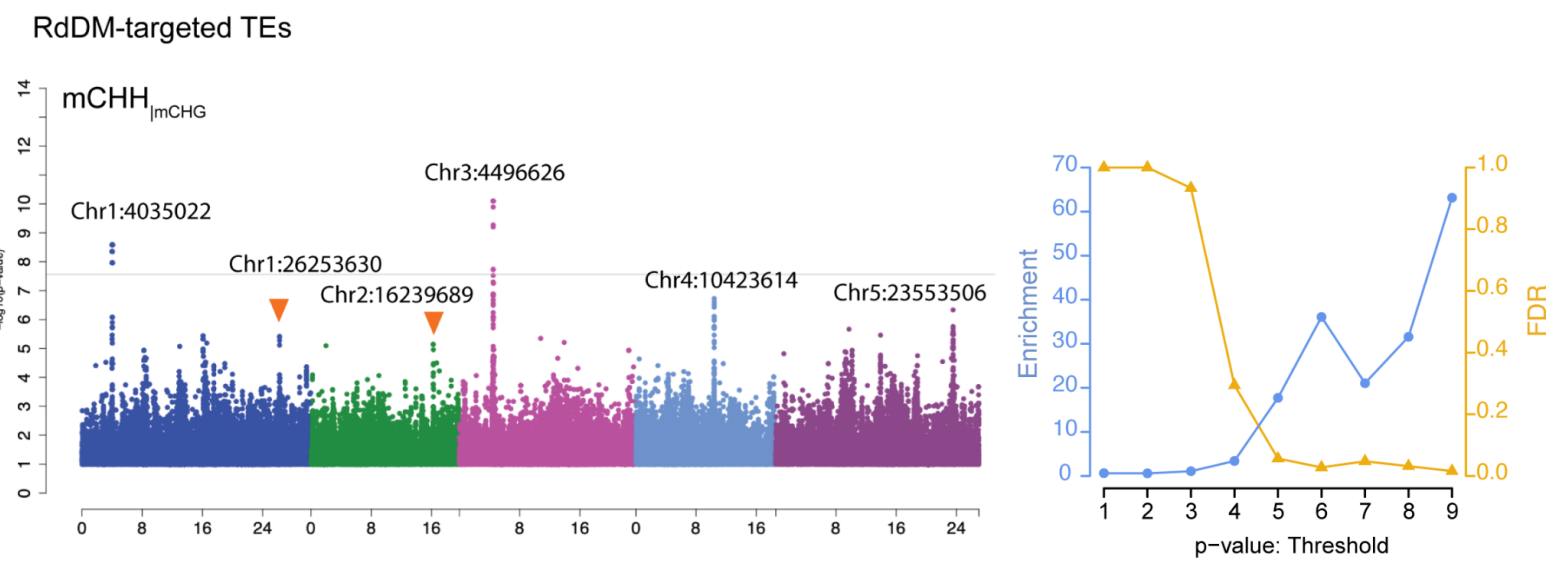

CMT2-targeted TEs
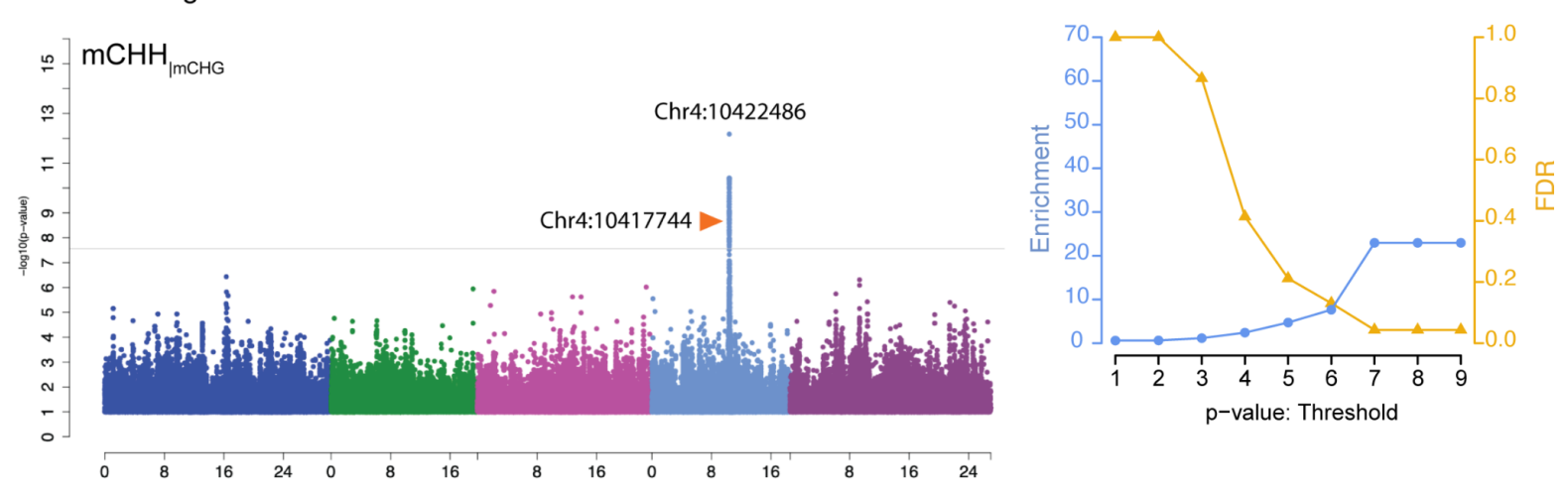

Fig S13. Conditional GWAS for $\mathrm{mCHH}_{\mid \mathrm{mcHG}}$. The genetic effects on $\mathrm{mCHH}$ in RdDM- and CMT2targeted transposons were analyzed by the conditional GWAS model with $\mathrm{mCHG}$ as cofactor. Vertical lines correspond to genome-wide significance ( $p=0.05$ by Bonferroni correction). Orange arrows indicate peaks reported in previous studies as affecting $\mathrm{mCHH}$ (Kawakatsu et al., 2016). Each GWAS result was assessed by enrichment of a priori genes and FDR. 